\title{
The expression of HOXA13 in lung adenocarcinoma and its clinical significance: A study based on The Cancer Genome Atlas, Oncomine and reverse transcription-quantitative polymerase chain reaction
}

\author{
YUN DENG $^{1 *}$, RONGQUAN HE ${ }^{2 *}$, RUI ZHANG ${ }^{1}$, BINLIANG GAN ${ }^{2}$, \\ YU ZHANG ${ }^{1},{\text { GANG } \mathrm{CHEN}^{1} \text { and XIAOHUA HU }}^{2}$ \\ Departments of ${ }^{1}$ Pathology and ${ }^{2}$ Medical Oncology, First Affiliated Hospital of Guangxi Medical University, \\ Nanning, Guangxi 530021, P.R. China
}

Received September 27, 2017; Accepted March 2, 2018

DOI: $10.3892 / \mathrm{ol} .2018 .8381$

\begin{abstract}
Previous studies have investigated the association between HOXA13 and non-small cell lung cancer. However, the role of HOXA13 expression in the occurrence and progression of lung adenocarcinoma (LUAD) has not yet been investigated. In the present study, HOXA13-related data mining of The Cancer Genome Atlas (TCGA), polymerase chain reaction (PCR) data from our cases and the case information in Oncomine was conducted for validation. The expression data of HOXA13 in lung cancer cell lines were also collected from the Cancer Cell Line Encyclopedia (CCLE) database for further verification. A comprehensive meta-analysis of the expression of HOXA13 was also performed, integrating the data of TCGA, in-house PCR and Oncomine. Genes that were co-expressed with HOXA13 were subsequently identified through cBioPortal and Multi Experiment Matrix (MEM), and the potential role and mechanism of HOXA13 in LUAD was investigated. The expression value of HOXA13 in the LUAD group, which comprised 237 cases, was $3.74 \pm 2.694$, significantly higher than its expression value in the non-cancerous group $(0.92 \pm 0.608, \mathrm{P}<0.001)$. The pooled SMD for HOXA13
\end{abstract}

Correspondence to: Professor Gang Chen, Department of Pathology, First Affiliated Hospital of Guangxi Medical University, 6 Shuangyong Road, Nanning, Guangxi 530021, P.R. China

E-mail: chen_gang_triones@163.com

Professor Xiaohua Hu, Department of Medical Oncology, First Affiliated Hospital of Guangxi Medical University, 6 Shuangyong Road, Nanning, Guangxi 530021, P.R. China

E-mail: gxmuhxh@163.com

*Contributed equally

Key words: HOXA13, lung adenocarcinoma, The Cancer Genome Atlas, reverse transcription-quantitative polymerase chain reaction, gene ontology, Kyoto Encyclopedia of Genes and Genomes was 0.346 (95\% CI, 0.052-0.640; $\mathrm{P}=0.068 ; \mathrm{I} 2=51.3 \%$; $\mathrm{P}=0.021$ ), The meta-analysis of diagnostic tests revealed that the area under the summary receiver operating characteristic curve (SROC) was 0.78 (95\% CI, 0.75-0.82). The results demonstrated that HOXA13 is highly expressed in LUAD. In addition to the studies on HOXA13 expression in tissues, the expression data of HOXA13 in lung cancer cell lines were also collected from the CCLE database for further verification of these conclusions. Genes that were co-expressed with HOXA13 were identified for pathway analysis. The most enriched Gene Ontology terms in the genes co-expressed with HOXA13 were positive regulation of transcription from RNA polymerase II promoter, signal transduction and positive regulation of GTPase activity in biological process; cytoplasm, integral component of membrane and plasma membrane in cellular component; and significantly involved in protein binding, transcription factor activity, sequence-specific DNA binding and sequence-specific DNA binding in molecular function. Kyoto Encyclopedia of Genes and Genomes analysis revealed that these target genes were clearly involved in Pathways in cancer, Proteoglycans in cancer and cAMP signaling pathway. The hub genes obtained from the four protein-protein interaction networks were associated with HOXA13. The results of the bioinformatics research in the present study revealed that HOXA13 may influence the expression of these hub genes in such a way as to promote the occurrence and development of LUAD. In conclusion, the expression of HOXA13 in patients with LUAD and its potential clinical value were analyzed comprehensively in the present study using data from a variety of sources. Through bioinformatics analysis, evidence that HOXA13 may promote the occurrence and development of LUAD was obtained.

\section{Introduction}

Lung cancer is the primary cause of mortality for patients with cancer globally, and the incidence of lung cancer ranks first among all malignancies in China and worldwide (1-4). Lung cancer includes small cell lung cancer and non-small 
cell lung cancer (NSCLC). Lung adenocarcinoma (LUAD) is a type of NSCLC and is one of the most common types of lung cancer. It accounts for 20-30\% of all types of primary lung cancer, and its incidence has surpassed that of squamous cell carcinoma in a number of countries (5-9). At present, comprehensive treatment of LUAD in clinical practice utilizes multiple methods, including surgical treatment, radiotherapy, chemotherapy and molecular targeted therapy. Recently, relatively rapid improvement has been made in the treatment of LUAD, but the prognosis and treatment efficacy of LUAD remains insufficient. The overall 5-year survival rate for all types of lung cancer, including LUAD, remains very low $(<15 \%)(10-14)$. Therefore, it is essential to expand and improve understanding of the principal molecular mechanisms associated with LUAD.

Hox genes, also known as homeotic genes or homologous genes, are genes that specifically regulate the biological morphology of living organisms. The mammalian Hox genes are categorized into four clusters, Hox A, B, C and D; these clusters are arranged on different chromosomes and are located at 7p15.3 (HoxA), 17q21.3 (HoxB), 12q13.3 (HoxC) and $2 \mathrm{q} 31$ (HoxD) in humans. HOXA13 is a member of the HOX family $(15,16)$. In recent years, the role of HOXA13 in cancer has been extensively investigated. It has been revealed that HOXA13 expression levels are associated with the occurrence of tumors of the digestive tract, gliomas, prostate cancer, cervical cancer, bladder cancer and ovarian cancer (17-24). There are currently only two studies on the role of HOXA13 in lung cancer. Sang et al (25) studied the association between the long non-coding RNAs (lncRNAs) HOTTIP and HOXA13 in NSCLC. Another study examined the critical genetic landmarks in early LUAD by utilizing high-density genomic arrays to detect increases in the copy numbers of genes located on the short arm of chromosome 7; that study led to the discovery of certain important genes that had not previously been identified, including HOXA13 (26). However, the role of HOXA13 expression in the occurrence and progression of LUAD has not been investigated. The prevalence of big data [comprising data from TCGA (which collected and characterized high-quality tumor and matched normal samples from over 11,000 patients) and Oncomine (which including 729 datasets and 91,866 samples)] has also provided us with a novel opportunity to study the clinical significance of HOXA13.

The present study conducted HOXA13-related data mining of The Cancer Genome Atlas (TCGA; http://cancergenome. nih.gov/) and used the polymerase chain reaction (PCR) data from our cases and the case information in Oncomine (www.oncomine.org) for validation. In order to obtain a full illustration of the expression of HOXA13 in tissue samples representing different detection methods, the data of TCGA, in-house PCR and Oncomine were integrated to perform a comprehensive meta-analysis. In addition to the HOXA13 expression in tissues, the expression data of HOXA13 in lung cancer cell lines were also collected from the Cancer Cell Line Encyclopedia (CCLE) database (http://www.broadinstitute. org/ccle) for further verification. Genes that were co-expressed with HOXA13 were subsequently identified through cBioPortal and Multi Experiment Matrix (MEM; http://biit.cs.ut .ee/mem/), and the potential role and mechanism of HOXA13 in LUAD was investigated.

\section{Materials and methods}

Extraction and analysis of data from the TCGA database. The raw data of rnaseqv2 in LUAD were downloaded from the TCGA database, including 237 cases of LUAD and 9 non-cancerous controls. Cases with expression values $<1$ were excluded. To further normalize the data, the HOXA13 expression values of the remaining cases were $\log 2$ transformed. The corresponding clinical parameters of the patients were subsequently extracted.

Validation of expression via oncomine. The Oncomine screening conditions were as follows: Gene name, HOXA13; analysis type, cancer vs. normal analysis; and data type, mRNA. Following screening, four datasets: 'Hou lung' (GSE19188, http://www.ncbi.nlm.nih. gov/geo/query/acc.cgi?acc=GSE19188) (27), 'Selamat Lung' (GSE32863, http://www.ncbi.nlm.nih.gov/geo/query/acc. cgi?acc=GSE32863)(28), 'GarberLung' (GSE3398, http://www. ncbi.nlm.nih.gov/geo/query/acc.cgi?acc=GSE3398) (29) and 'Okayama Lung' (GSE31210, http://www.ncbi.nlm.nih. gov/geo/query/acc.cgi?acc=GSE31210) (30) were included.

Validation using reverse transcription-quantitative PCR data of the clinical samples in our hospital. Paraffin-embedded LUAD specimens from patients that had undergone pneumonectomy at the First Affiliated Hospital of Guangxi Medical between January 2012 and February 2014 were collected and pathologically confirmed. A total of 29 cases with complete data were selected, including 20 males and 9 females; the ages of the patients were 23-90 years, mean age, 57 years. Lung cancer tissues and corresponding non-cancerous tissues were obtained from each patient. All specimens were removed, fixed for $48 \mathrm{~h}$ in $10 \%$ formaldehyde at room temperature, and the thickness of the specimens were $3 \mathrm{~mm}$; they were subjected to routine embedding in paraffin. The present study was approved by the Ethical Committee of the First Affiliated Hospital of Guangxi Medical University, and all patients provided written informed consent for participation in the study. Total RNA was extracted from FFPE tissues using the RNeasy reagent (Qiagen China Co., Ltd., Shanghai, China) and was reverse transcribed in a final volume of $20 \mu \mathrm{l}$ with random primers using the PrimeScript RT reagent kit (Takara Biotechnology Co., Ltd., Dalian, China), according to the manufacturer's protocols. SYBR Premix Ex Taq (Takara Biotechnology Co., Ltd.) was used to detect HOXA13 expression levels. The results were normalized to the expression of the internal reference gene, GAPDH, and calculated using the $2^{-\Delta c t}$ method (31) according to the manufacturer's protocols of the Applied Biosystems Fast Real-Time PCR System (Applied Biosystems; Thermo Fisher Scientific, Inc., Waltham, MA, USA). The PCR procedure was: Initial denaturation at $95^{\circ} \mathrm{C}$ for $10 \mathrm{~min}$, denaturation at $95^{\circ} \mathrm{C}$ for $10 \mathrm{sec}$; refolding for $5 \mathrm{sec}$ at annealing temperature $60^{\circ} \mathrm{C}$; extension at $72^{\circ} \mathrm{C}$ for $5 \mathrm{sec}$ (total 40 cycles). The primer sequences were as follows: HOXA13, forward 5'-GAACGGCCAAAT GTACTGCC-3', reverse 5'-CGCCTCCGTTTGTCCTTA GT-3. GAPDH, forward 5'-TGCACCACCAACTGCTTA-3' reverse 5'-GGATGCAGGGATGATGTTC-3' (25). 
Validation using cell line data from CCLE. 'HOXA13' was searched in the CCLE database and the expression data of HOXA13 from all the cancer cell lines was downloaded. A total of 192 lung cancer cell lines were selected. The PC-14 cell line has been reported to be contaminated or misidentified in International Cell Line Authentication Committee, Database of Cross-Contaminated or Misidentified Cell Lines (http://iclac.org/databases/cross-contaminations/), and was therefore excluded from the present study. A total of 191 cell lines were collected for further research. A heat-map based on the expression of HOXA13 in different lung cancer cell lines was created by HemI (Heatmap Illustrator, version 1.0; http://hemi.biocuckoo.org/). These 191 cell lines exhibited varying degrees of high (red) or low (blue) expression of HOXA13.

Statistical analysis. Data analysis was conducted with SPSS 24.0 software (IBM Corp., Chicago, IL, USA), and plots were created with GraphPad Prism 7 software (GraphPad Software, San Diego, CA, USA). The relative expression of HOXA13 is presented as the mean \pm standard deviation. An independent Student's t-test was applied for the comparison of cancerous and non-cancerous samples, and paired t-text was used to comparison of cancerous and non-cancerous samples of PCR, one-way analysis of variance was used for the comparison of three groups and LDS-t test was used as a post hoc test. Correlations were analyzed by Spearman's correlation analysis. The associations between HOXA13 expression and the main clinicopathological characteristics of patients with LUAD were analyzed using the independent sample t-test, and alterations in expression between two groups were demonstrated by scatter plots. The diagnostic value of HOXA13 for LUAD was analyzed using the receiver operating characteristic (ROC) curve. The area under the curve (AUC) was calculated, and the Youden index [Youden index=sensitivity-(1-specificity)] was used to assess the optimal diagnostic threshold. The standard mean difference (SMD) with 95\% CI was calculated using STATA 12.0 (StataCorp, College Station, TX, USA). If SMD $>0$ and its 95\% CI does not cross the 0 value, it indicates that expression of HOXA13 in tumors is higher than in the non-cancerous tissues. Summary receiver operating characteristic (SROC) curve, sensitivity (SEN), specificity (SPE), positive likelihood ratio (PLR), negative likelihood ratio (NLR), diagnostic score (DS) and diagnostic odds ratio (DOR) were calculated using STATA 12.0. I2 test was used to test heterogeneity between each of the studies. It was considered that there would exist heterogeneity between studies when P-values were less than 0.05 or I 2 values were more than $50 \%$. The random-effects model or the fixed-effects model were used to synthesize the data. The correlations between HOXA13 and clinicopathological features and the expression of related genes were calculated using Pearson's correlation analysis. $\mathrm{P}<0.05$ was considered to indicate a statistically significant difference.

Prediction of related genes. In MEM, the output option for each probe was set to 1,500 and the resulting similarity genes were then exported. Genes that were present in at least two probe groups were selected. cBioPortal (http://www .cbioportal.org/index.do) incorporates data from 126 tumor genome research projects, including TCGA, International Cancer Genome Consortium and other large tumor research projects, and includes data from 28,000 cases. The co-expression analysis module of cBioPortal is able to extract genes that are co-expressed with HOXA13; co-expressed genes are defined as genes associated with HOXA13 expression. The associated genes that were identified by MEM and cBioPortal were subjected to follow-up pathway analysis.

Gene enrichment and functional annotation evaluation. The Database for Annotation, Visualization, and Integrated Discovery (DAVID; http://david.abcc.ncifcrf.gov/) was used to conduct relevant pathway analysis, and Gene Ontology (GO) analysis was performed for the functional annotation of the co-expressed genes. Three GO terms [biological process (BP), cellular component (CC) and molecular function (MF)] were utilized to identify the enrichment of target genes. GO terms and KEGG pathways with P-values $<0.05$ were considered statistically significant. The enrichment map of annotation analysis was drawn using Cytoscape version 3.3.0 (http://www .cytoscape.org/cy3.html).

The protein-protein interaction (PPI) network. The genes involved in the first four pathways of the KEGG pathway analysis were selected for PPI analysis. Four PPI networks were established through a bioinformatics platform called STRING (http://www.string-db.org). The PPI data were downloaded from the STRING database, and a map of the complete PPI network was created. Hub genes, which may be recognized as highly connected genes in the network, were identified according to the value of degrees of each node. Genes with the first and second values of degrees in each PPI network were considered to be hub genes.

Correlation between HOXA13 and hub genes. The $\log 2$ values of the expression values of HOXA13 and hub genes in the TCGA data were used for correlation analysis. GraphPad Prism version 5.0 (GraphPad Software, Inc.) was utilized to create the association diagram between hub genes and HOXA13 expression in the TCGA database, and scatter plots of hub gene expression in non-cancerous tissues and LUAD tissues based on the data from the TCGA database. The differences in the expression of hub genes in LUAD tissues and non-cancerous tissues were analyzed using the ROC curve. The AUC value was between 0.5 and 1.0.

\section{Results}

Expression of HOXA13 in the TCGA database. The expression value of HOXA13 in the LUAD group, which comprised 237 cases, was $3.74 \pm 2.694$, significantly higher than its expression value in the non-cancerous group $(0.92 \pm 0.608 ; \mathrm{P}<0.001$; Fig. 1Aa and Table I). The expression value of HOXA13 in the LUAD group was 4 times its value in the non-cancerous group. The diagnostic value of HOXA13 was calculated based on the ROC curve; the AUC value was 0.839 (95\% confidence interval $(\mathrm{CI}), 0.765-0.913 ; \mathrm{P}=0.001$; Fig. $1 \mathrm{Ab})$. The cut-off value of HOXA13 expression was 1.27 . The sensitivity was $76.4 \%$ and the specificity was $88.9 \%$. 

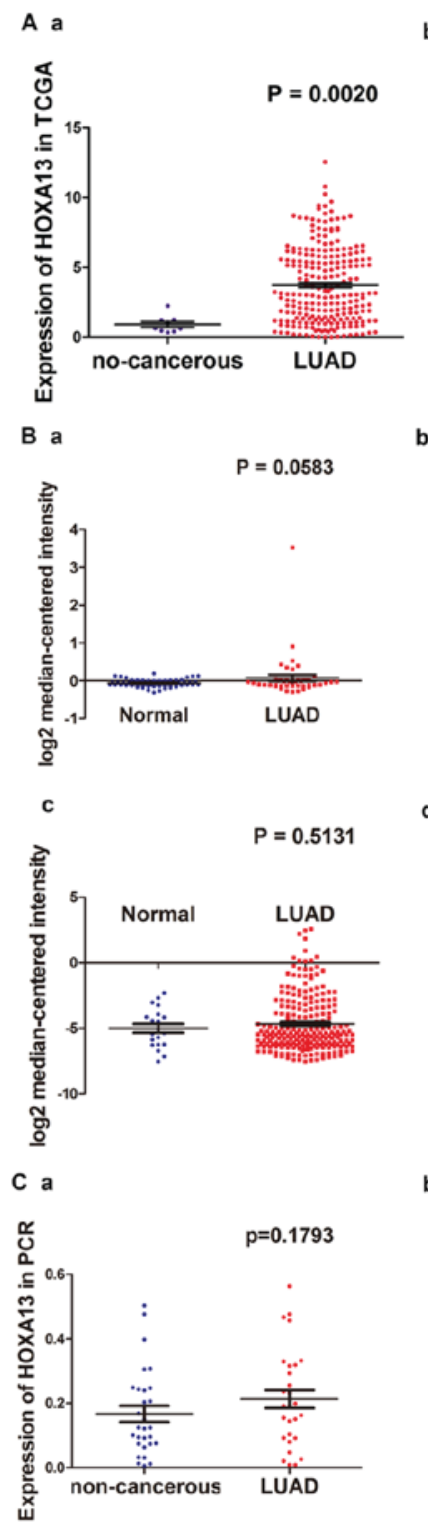

c

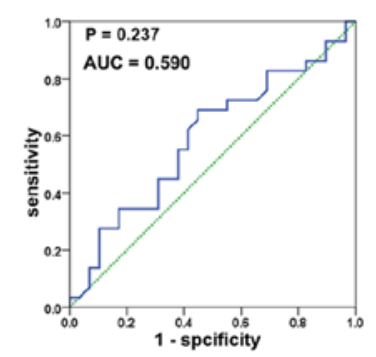

b

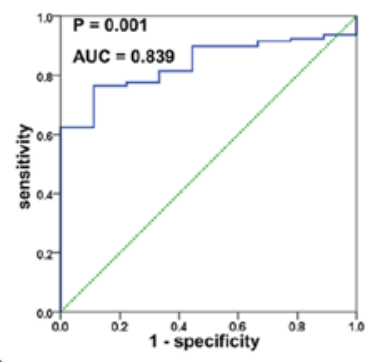

b
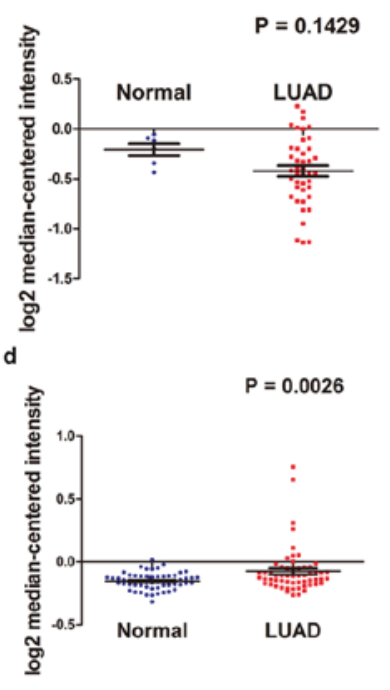

b

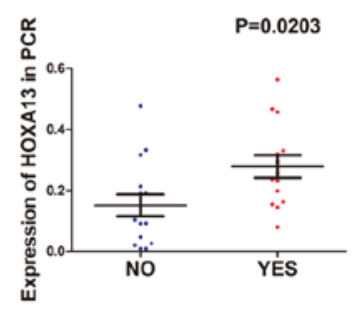

Figure 1. (A) Expression of HOXA13 in the TCGA database. (Aa) The expression value of HOXA13 in the LUAD group and the non-cancerous group in the TCGA database; $(\mathrm{Ab})$ ROC curve for discrimination of LUAD tissues from non-cancerous tissues (non-cancerous, 9 cases; LUAD, 237 cases). (B) Validation of HOXA13 expression based on Oncomine. The difference in HOXA13 expression between patients with LUAD and healthy individuals. (Ba) 'Hou Lung' (normal, 65 cases; LUAD, 45 cases); (Bb) 'Garber Lung' (normal, 6 cases; LUAD, 42 cases); (Bc) 'Okayama Lung' (normal, 20 cases; LUAD, 226 cases); (Bd) 'Selamat Lung' (normal, 58 cases; LUAD, 58 cases). (C) Validation based on reverse transcription-quantitative PCR results of the clinical samples obtained at our hospital. (Ca) Distinguish LUAD from non-cancerous tissues (normal, 29 cases; lung adenocarcinoma, 29 cases); (Cb) lymph node metastasis (No, 15 cases; Yes, 14 cases); (Cc) ROC curve for discrimination of LUAD from non-cancerous tissues. TCGA, The Cancer Genome Atlas; LUAD, lung adenocarcinoma; ROC, receiver operating characteristic; AUC, area under the curve; PCR, polymerase chain reaction.

Validation of HOXA13 expression based on oncomine. A total of four sets of data were obtained from Oncomine, including 'Hou Lung' (GSE19188, normal, 65 cases; LUAD, 45 cases), 'Selamat Lung' (GSE32863, normal, 58 cases; LUAD, 58 cases), 'Garber Lung' (GSE3398, normal, 6 cases; LUAD, 42 cases) and 'Okayama Lung' (GSE31210, normal, 20 cases; LUAD, 226 cases). In the study undertaken by Hou et al (GSE19188) (27), a particular trend was observed and the relative expression of HOXA13 was higher in the LUAD tissue group than in the normal lung tissue group (Fig. 1Ba), but this difference was not significant $(\mathrm{P}=0.0583)$. The expression value of HOXA13 was significantly higher in the LUAD tissue group compared with the normal lung tissue group in the study undertaken by Selamat et al (GSE32863; Fig. 1Bd; $\mathrm{P}=0.0026$ ) (28), The expression of HOXA13 was lower in the LUAD cases compared with normal cases in the study undertaken by Garber et al (GSE3398; Fig. 1Bb; P=0.1429) (29). The opposite situation was demonstrated in the study undertaken by Okayama et al (GSE31210; Fig. 1Bc; $\mathrm{P}=0.5131)$ (30), consistent with the TCGA data obtained in the present study.

Validation based on RT-qPCR results of the clinical samples obtained at our hospital. The expression of HOXA13 in the LUAD group (29 cases) was $0.21 \pm 0.150$, slightly higher than that in the non-cancerous group $(0.17 \pm 0.134$; Fig. $1 \mathrm{Ca})$, but this difference was not statistically significant. The expression value of HOXA13 in the cancerous tissues was 1.3 times that in the non-cancerous tissues. HOXA13 was predominantly overexpressed in patients with lymph node metastasis, compared with expression in the non-metastatic group $(0.28 \pm 0.138$ vs. $0.15 \pm 0.138 ; \mathrm{P}=0.020$; Fig. $1 \mathrm{Cb}$ and Table II). HOXA13 expression was not significantly correlated with other clinical parameters (Table II). The present study also calculated the diagnostic value of HOXA13 for LUAD; the results demonstrated that the AUC was 0.590 (95\% CI, 0.442-0.739; $\mathrm{P}=0.237$; Fig. 1Cc). The cut-off value for HOXA13 was 0.13 . The sensitivity was $69 \%$ and the specificity was $55.2 \%$.

Validation using cell line data from CCLE. A total of 191 lung cancer cell lines were selected. Each cell line had a corresponding value of HOXA13 expression. The heat-map for expression of HOXA13 in the lung cancer cell lines revealed that more than half of the bands were red, indicating overexpression of HOXA13 (Fig. 2; Table III).

Meta-analyses. To obtain a comprehensive result of HOXA13 expression in LUAD, data from TCGA, Oncomine and in-house PCR were integrated, and a meta-analysis was performed. Using the random-effects model, the pooled SMD for HOXA13 was 0.346 (95\% CI, 0.052-0.640; $\mathrm{P}=0.068$; $\mathrm{I} 2=51.3 \%$; $\mathrm{P}=0.021$; Fig. 3A). The meta-analyses of diagnostic tests revealed that the AUC for the SROC of HOXA13 in LUAD was 0.78 (95\% CI, 0.75-0.82; Fig. 3B). The pooled SEN, SPE, PLR, NLR, DS and DOR of HOXA13 in these studies were 0.64 (95\%CI, 0.31-0.88), 0.77 (95\% CI, 0.55-0.90), 2.81 (95\% CI, 1.72-4.58), 0.46 (95\% CI, 0.22-0.97), 1.80 (95\% CI, 0.92-2.68) and 6.05 (95\% CI, 2.52-14.54), respectively (Fig. 3C-H). The aforementioned results demonstrated that HOXA13 was highly expressed in LUAD, based on 6 independent studies and 826 cases. 
Table I. Expression of HOXA13 in the TCGA database.

HOXA13 expression in TCGA

\begin{tabular}{|c|c|c|c|c|}
\hline \multirow{2}{*}{ Clinicopathological feature } & \multirow[b]{2}{*}{$\mathrm{N}$} & & & \\
\hline & & Mean \pm SD & $\mathrm{T}$ or $\mathrm{F}$ & P-value \\
\hline \multicolumn{5}{|l|}{ Tissue } \\
\hline Lung adenocarcinoma & 237 & $3.74 \pm 2.694$ & \multirow[t]{2}{*}{10.527} & \multirow[t]{2}{*}{$<0.001$} \\
\hline Non-cancerous & 9 & $0.92 \pm 0.608$ & & \\
\hline \multicolumn{5}{|l|}{ Age, years } \\
\hline$<60$ & 72 & $3.48 \pm 2.488$ & \multirow[t]{2}{*}{-0.96} & \multirow[t]{2}{*}{0.337} \\
\hline$\geq 60$ & 165 & $3.85 \pm 2.779$ & & \\
\hline \multicolumn{5}{|l|}{ Sex } \\
\hline Male & 105 & $3.77 \pm 2.746$ & \multirow[t]{2}{*}{0.196} & \multirow{2}{*}{0.845} \\
\hline Female & 132 & $3.70 \pm 2.662$ & & \\
\hline \multicolumn{5}{|l|}{ Ethnicity } \\
\hline White & 184 & $3.79 \pm 2.707$ & \multirow[t]{3}{*}{$\mathrm{F}=1.318$} & \multirow[t]{3}{*}{0.270} \\
\hline Black & 21 & $2.96 \pm 2.223$ & & \\
\hline Asian & 2 & $5.39 \pm 0.069$ & & \\
\hline \multicolumn{5}{|l|}{ T stage } \\
\hline $\mathrm{T} 1+\mathrm{T} 2$ & 202 & $3.74 \pm 2.672$ & \multirow[t]{2}{*}{-0.093} & \multirow[t]{2}{*}{0.926} \\
\hline $\mathrm{T} 3+\mathrm{T} 4$ & 33 & $3.78 \pm 2.874$ & & \\
\hline \multicolumn{5}{|l|}{$\mathrm{N}$} \\
\hline NX & 7 & $1.97 \pm 1.771$ & \multirow[t]{3}{*}{2.647} & \multirow[t]{3}{*}{0.073} \\
\hline N0-N1 & 188 & $3.67 \pm 2.677$ & & \\
\hline N2-N3 & 42 & $4.34 \pm 2.775$ & & \\
\hline \multicolumn{5}{|l|}{ M } \\
\hline MX & 67 & $4.05 \pm 2.569$ & \multirow[t]{3}{*}{0.689} & \multirow[t]{3}{*}{0.503} \\
\hline M0 & 155 & $3.63 \pm 2.680$ & & \\
\hline M1 & 12 & $4.11 \pm 3.477$ & & \\
\hline \multicolumn{5}{|l|}{ Stage } \\
\hline I+II & 142 & $3.58 \pm 2.621$ & \multirow[t]{2}{*}{-1.160} & \multirow[t]{2}{*}{0.247} \\
\hline III+IV & 94 & $3.99 \pm 2.808$ & & \\
\hline Status & & & & \\
\hline Deceased & 101 & $3.86 \pm 2.876$ & 0.600 & 0.549 \\
\hline Living & 136 & $3.64 \pm 2.557$ & & \\
\hline Recurrence & & & & \\
\hline Distant metastasis & 42 & $3.86 \pm 2.561$ & 2.780 & 0.069 \\
\hline Loco-regional recurrence & 24 & $2.92 \pm 2.207$ & & \\
\hline New primary tumor & 2 & $0.39 \pm 0.486$ & & \\
\hline Person neoplasm cancer stat & & & & \\
\hline With tumor & 87 & $3.68 \pm 2.706$ & -0.212 & 0.833 \\
\hline Tumor-free & 123 & $3.76 \pm 2.623$ & & \\
\hline
\end{tabular}

TCGA, The Cancer Genome Atlas; SD, standard deviation; T, T-value (Student's t-test); F, F-value (one-way analysis of variance); TNM, Tumor-Node-Metastasis.

Prediction of related genes and gene-enrichment and functional annotation analyses. There were 20,437 genes co-expressed with HOXA13 in cBioPortal and 717 genes co-expressed with HOXA13 in MEM. A total of 679 genes were obtained in the intersection of the aforementioned two groups of co-expressed genes. The significantly enriched biological terms were identified by their P-values of less than 0.05 (Table III). The results demonstrated that the target genes were most highly enriched in the following biological pathways: Steroid hormone-mediated signaling pathway, positive regulation of transcription from RNA polymerase II promoter, and negative regulation of transcription, DNA-templated $(\mathrm{P}<0.001$; Table IV; Figs. 4 and 5). KEGG pathway analysis results demonstrated that the 
Table II. Differential expression of HOXA13 in LUAD tissues based on in-house reverse transcription-quantitative polymerase chain reaction.

\begin{tabular}{|c|c|c|c|c|}
\hline \multirow[b]{2}{*}{ Clinicopathological feature } & \multirow[b]{2}{*}{$\mathrm{N}$} & \multicolumn{3}{|c|}{ HOXA13 expression $\left(2^{-\Delta c t}\right)$} \\
\hline & & Mean \pm SD & $\mathrm{T}$ or $\mathrm{F}$ & P-value \\
\hline \multicolumn{5}{|l|}{ Tissues } \\
\hline Non-cancerous & 29 & $0.17 \pm 0.134$ & 1.229 & 0.224 \\
\hline LUAD & 29 & $0.21 \pm 0.150$ & & \\
\hline \multicolumn{5}{|l|}{ Size, $\mathrm{cm}$} \\
\hline$\leq 3$ & 8 & $0.22 \pm 0.166$ & 0.167 & 0.868 \\
\hline$>3$ & 21 & $0.21 \pm 0.148$ & & \\
\hline \multicolumn{5}{|l|}{ TNM stage } \\
\hline I-II & 16 & $0.19 \pm 0.158$ & -1.011 & 0.321 \\
\hline III-IV & 13 & $0.24 \pm 0.139$ & & \\
\hline \multicolumn{5}{|l|}{$\operatorname{Sex}$} \\
\hline Male & 20 & $0.21 \pm 0.165$ & -0.301 & 0.766 \\
\hline Female & 9 & $0.23 \pm 0.119$ & & \\
\hline \multicolumn{5}{|l|}{ Age, years } \\
\hline$<60$ & 19 & $0.23 \pm 0.156$ & 0.938 & 0.357 \\
\hline$\geq 60$ & 10 & $0.18 \pm 0.139$ & & \\
\hline \multicolumn{5}{|l|}{ Smoking } \\
\hline No & 18 & $0.24 \pm 0.144$ & 1.455 & 0.157 \\
\hline Yes & 11 & $0.16 \pm 0.153$ & & \\
\hline \multicolumn{5}{|l|}{ EGFR mutation } \\
\hline Wild-type & 16 & $0.20 \pm 0.171$ & -0.520 & 0.607 \\
\hline Mutation & 12 & $0.23 \pm 0.130$ & & \\
\hline \multicolumn{5}{|l|}{ EGFR } \\
\hline No & 18 & $0.20 \pm 0.157$ & -0.444 & 0.661 \\
\hline Yes & 10 & $0.23 \pm 0.151$ & & \\
\hline \multicolumn{5}{|l|}{ Vascular invasion } \\
\hline No & 28 & $0.21 \pm 0.152$ & 0.413 & 0.683 \\
\hline Yes & 1 & $0.15 \pm 0$ & & \\
\hline \multicolumn{5}{|l|}{ LNM } \\
\hline No & 15 & $0.15 \pm 0.138$ & -2.467 & 0.020 \\
\hline Yes & 14 & $0.28 \pm 0.138$ & & \\
\hline \multicolumn{5}{|l|}{ EGFR protein } \\
\hline Low & 19 & $0.20 \pm 0.141$ & -0.680 & 0.502 \\
\hline High & 9 & $0.24 \pm 0.181$ & & \\
\hline \multicolumn{5}{|l|}{ MET } \\
\hline Low & 16 & $0.20 \pm 0.150$ & -0.550 & 0.587 \\
\hline High & 12 & $0.23 \pm 0.161$ & & \\
\hline \multicolumn{5}{|l|}{ Grading } \\
\hline I & 5 & $0.19 \pm 0.082$ & $\mathrm{~F}=0.151$ & 0.860 \\
\hline II & 21 & $0.22 \pm 0.172$ & & \\
\hline III & 3 & $0.18 \pm 0.062$ & & \\
\hline
\end{tabular}

LUAD, lung adenocarcinoma; SD, standard deviation; T, T-value (Student's t-test); F, F-value (one-way analysis of variance); TNM, Tumor-Node-Metastasis; EGFR, epidermal growth factor receptor; LNM, lymph node metastasis; MET, mesenchymal-to-epithelial transition factor. Grading: American Joint Committee on Cancer (AJCC) Cancer Staging Manual was used as grading system. GX: Grade cannot be assessed (undetermined grade), G1: Well differentiated (low grade), G2: Moderately differentiated (intermediate grade), G3: Poorly differentiated (high grade), G4: Undifferentiated (high grade). Group I contained GX, group II contained G1 and G2, G3 and G4 were included in group III. 


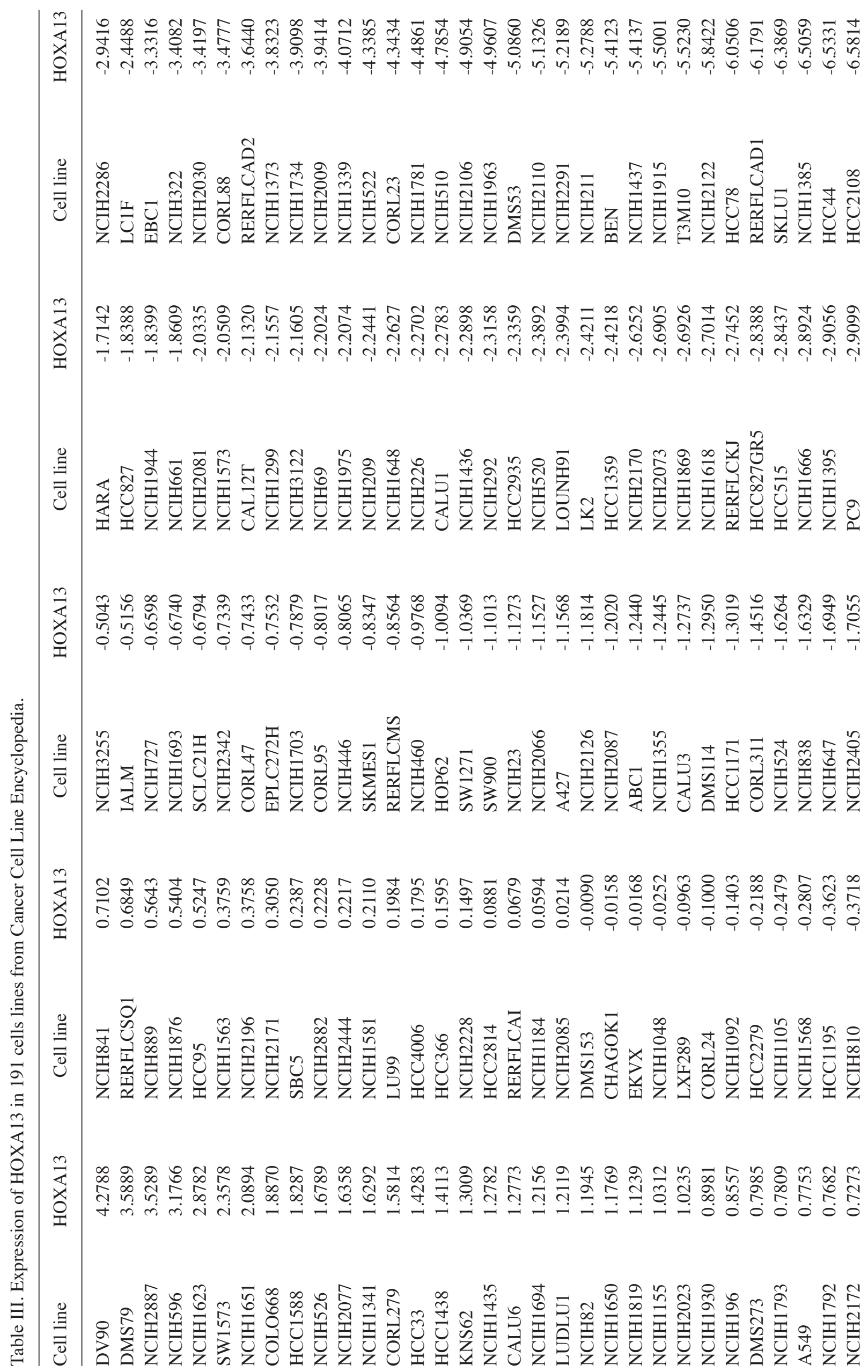



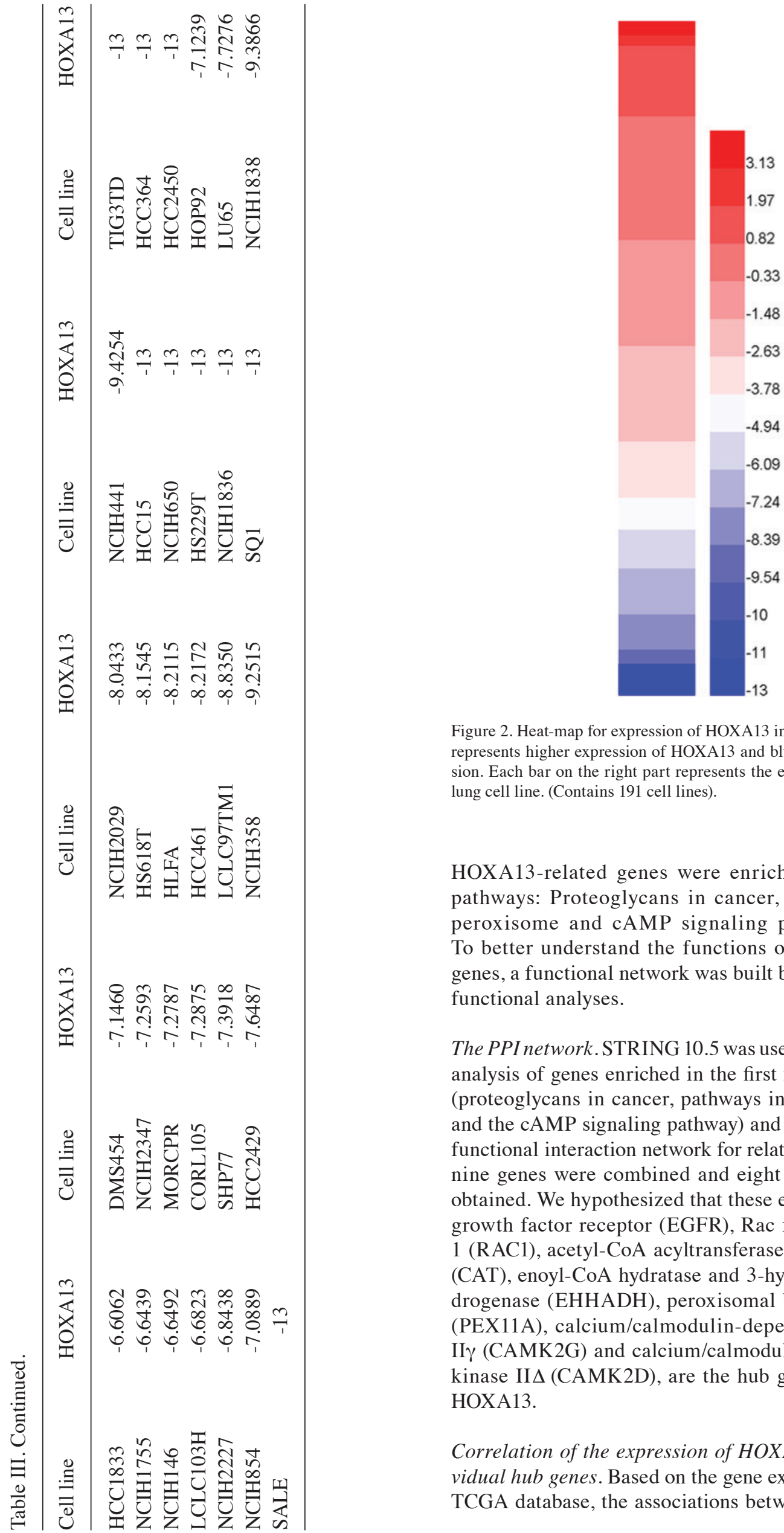

Figure 2. Heat-map for expression of HOXA13 in lung cancer cell lines. Red represents higher expression of HOXA13 and blue represents lower expression. Each bar on the right part represents the expression of HOXA13 in a lung cell line. (Contains 191 cell lines).

HOXA13-related genes were enriched in the following pathways: Proteoglycans in cancer, pathways in cancer, peroxisome and cAMP signaling pathway (Table IV). To better understand the functions of these co-expressed genes, a functional network was built based on the results of functional analyses.

The PPI network. STRING 10.5 was used to conduct an online analysis of genes enriched in the first four KEGG pathways (proteoglycans in cancer, pathways in cancer, peroxisomes and the cAMP signaling pathway) and to construct a protein functional interaction network for related genes (Fig. 6). The nine genes were combined and eight different genes were obtained. We hypothesized that these eight genes, epidermal growth factor receptor (EGFR), Rac family small GTPase 1 (RAC1), acetyl-CoA acyltransferase 1 (ACAA1), catalase (CAT), enoyl-CoA hydratase and 3-hydroxyacyl CoA dehydrogenase (EHHADH), peroxisomal biogenesis factor $11 \alpha$ (PEX11A), calcium/calmodulin-dependent protein kinase II $\gamma$ (CAMK2G) and calcium/calmodulin-dependent protein kinase II $\Delta$ (CAMK2D), are the hub genes associated with HOXA13.

Correlation of the expression of HOXA13 with that of individual hub genes. Based on the gene expression values in the TCGA database, the associations between the expression of 


\section{A}

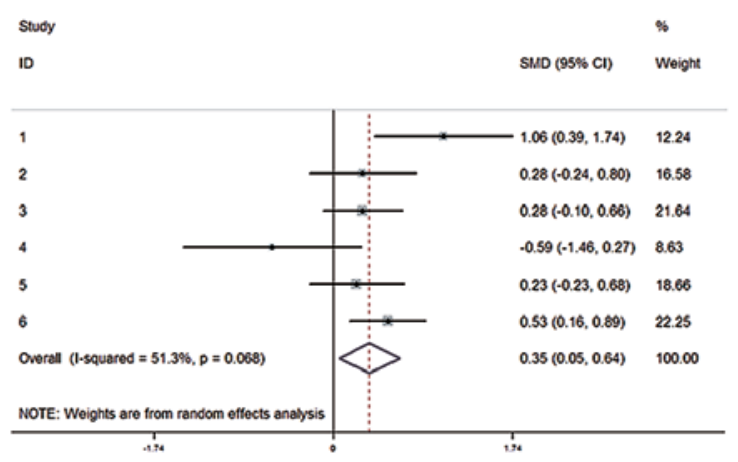

C

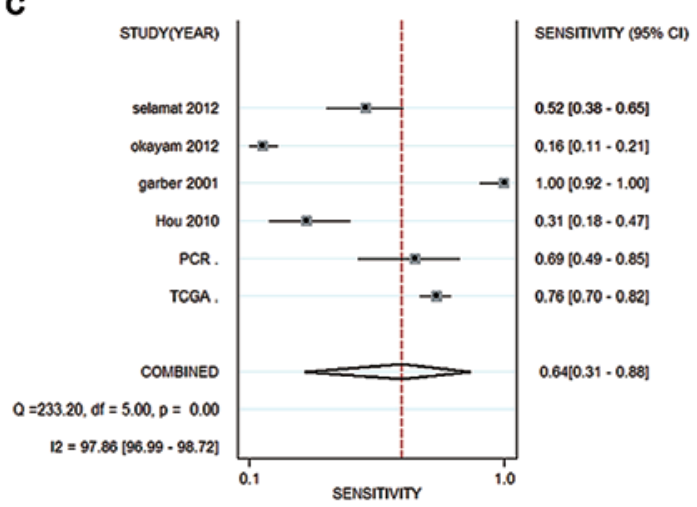

E

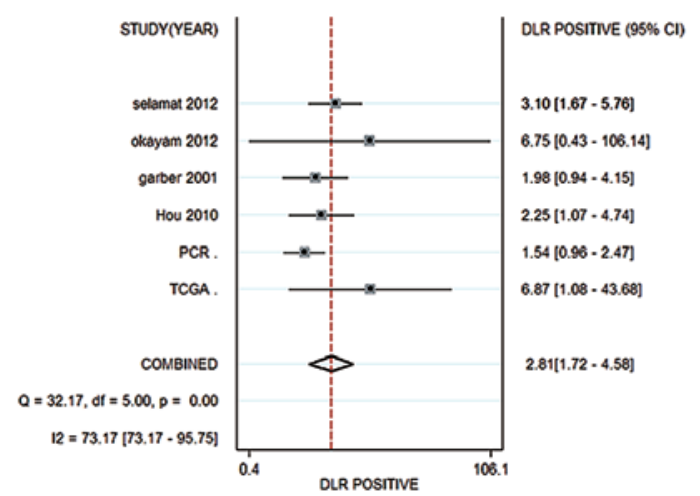

G

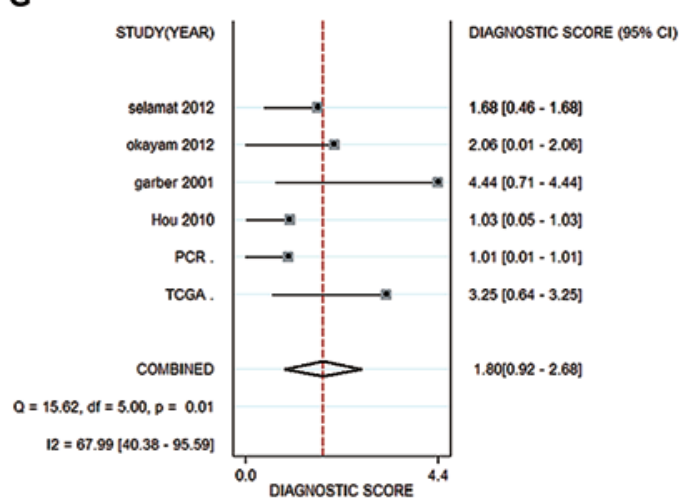

B

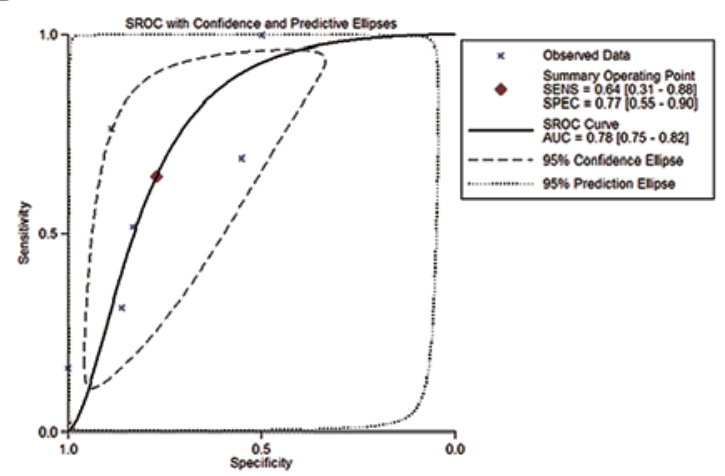

D

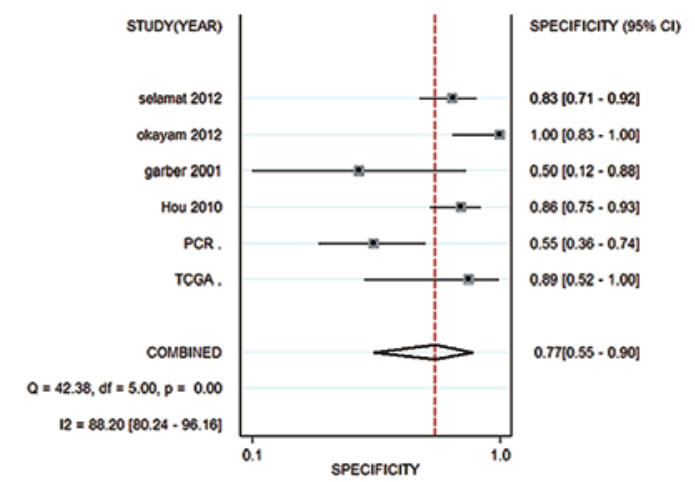

$\mathbf{F}$

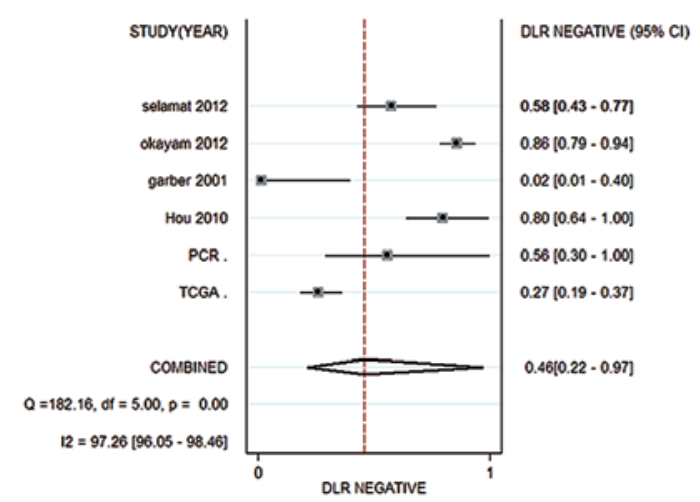

H

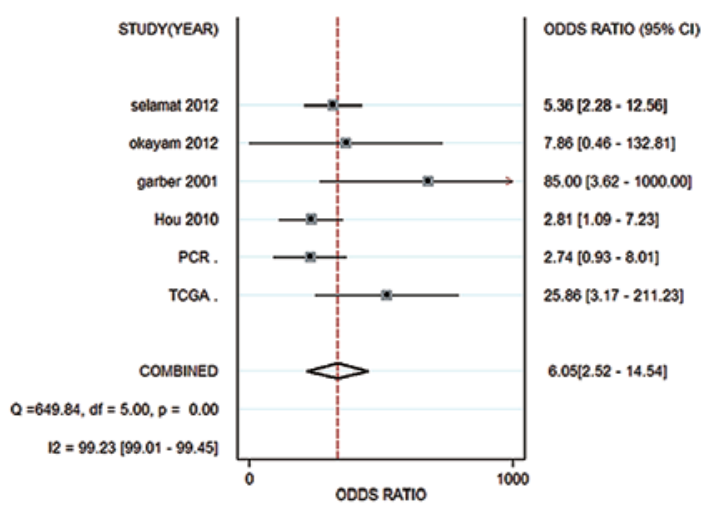

Figure 3. Meta-analysis. (A) Forest plot of meta-analyses of the diagnostic value of HOXA13 expression for patients with LUAD with six datasets involved. Random effects model was applied when combining SMD. (B) SROC curve for HOXA13 expression for patients with LUAD with six datasets involved. Each solid circle represents a study. The size of the solid circle represents the sample size of each eligible study. The overall diagnostic efficiency was summarized by the regression curve. (C) Forest plot of sensitivity for HOXA13 in LUAD. (D) Forest plot of specificity for HOXA13 in LUAD. (E) Forest plot of PLR for HOXA13 in LUAD. (F) Forest plot of NLR for HOXA13 in LUAD. (G) DS for HOXA13 in LUAD. (H) Diagnostic OR for HOXA13 in LUAD. Each solid circle represents a study. The size of the solid circle reflects the sample size of each study; the error bars represent the 95\% CI. LUAD, lung adenocarcinoma; SMD, standardized mean difference; SROC, summary receiver operating characteristic; AUC, area under the curve; SE, standard error; PLR, positive likelihood ratio; NLR, negative likelihood ratio; DS, diagnostic score; OR, odds ratio; CI, confidence intervals, SENS, sensitivity; SPEC, specificity; PCR, polymerase chain reaction. 


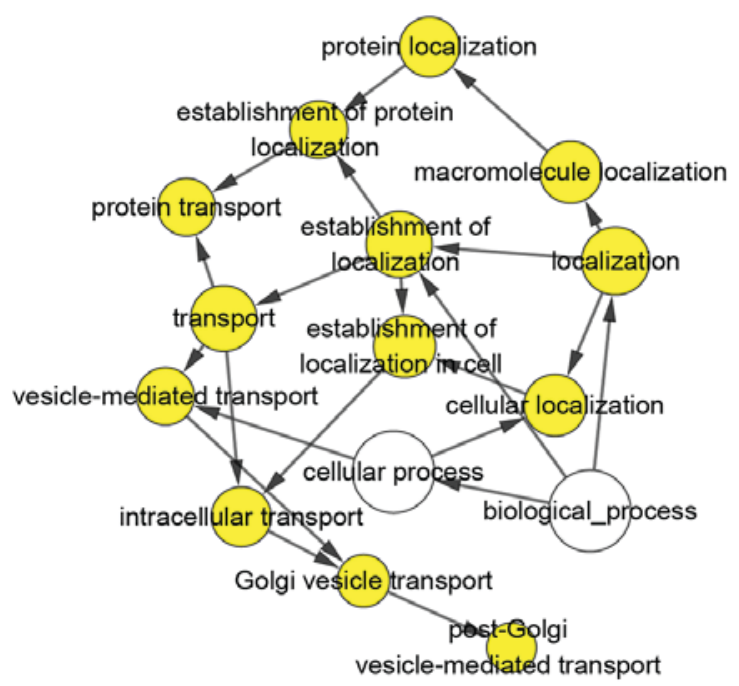

Figure 4. Gene Ontology analysis of Biological Process category analysis of HOXA13-related genes. Each circle represents a biological process in which related genes of HOXA13 participate.

HOXA13 and that of each of the eight hub genes in the tissues of patients with LUAD were verified. HOXA13 expression was significantly positively correlated with the expression of three of the eight hub genes: RAC1 ( $\mathrm{r}=0.2638$; $\mathrm{P}<0.0001)$, EHHADH ( $\mathrm{r}=0.2709 ; \mathrm{P}<0.0001)$ and PEX11A ( $\mathrm{r}=0.1274$; $\mathrm{P}=0.0426$ ). The correlation between HOXA13 expression and that of the other hub genes was not statistically significant (Fig. 7).

The expression of hub genes in the TCGA database. Expression of hub genes in normal and LUAD cases were as follows (normal, 59 cases; LUAD, 535 cases): Expression of EGFR in LUAD cases was lower compared with normal cases $(\mathrm{P}=0.5082$; Fig. $8 \mathrm{~A})$, the opposite was observed in RAC1 $(\mathrm{P}=0.0058$; Fig. 8B). Expression of ACAA1 in LUAD cases was lower compared with normal cases $(\mathrm{P}<0.0001$; Fig. 8C) and the same trend was observed in CAT $(\mathrm{P}<0.0001$; Fig. 8D). Expression of EHHADH in LUAD cases was higher compared with normal cases $(\mathrm{P}=0.5131$; Fig. $8 \mathrm{E})$ and the opposite result was identified in PEX11A $(\mathrm{P}=0.0003$; Fig. 8F). CAMK2G demonstrated a higher expression level in LUAD cases compared with normal cases $(P=0.1305$; Fig. 8G) and the opposite result was observed in CAMK2D $(\mathrm{P}=0.7277$; Fig. $8 \mathrm{H})$ The ROC curve of each of the hub genes was also calculated; the results demonstrated that the AUC of EGFR was 0.532 (95\% CI, 0.482-0.583; $\mathrm{P}=0.415)$, the cut-off value for EGFR was 12.33, and the sensitivity and specificity were 83.1 and $42.6 \%$, respectively. The AUC of RAC1 was 0.633 (95\% CI, 0.581-0.685; P=0.001), the cut-off value for RAC1 was 13.84 , and the sensitivity and specificity were 55 and $78 \%$, respectively. The AUC of ACAA1 was 0.700 (95\% CI, 0.65-0.75; $\mathrm{P}=0.0001$ ), the cut-off value for ACAA1 was 10.96 , and the sensitivity and specificity were 91.5 and $48.2 \%$, respectively. The AUC of CAT was 0.989 (95\% CI, 0.982-0.996; $\mathrm{P}=0.0001$ ), the cut-off value for CAT was 13.2 , and the sensitivity and specificity were 98.3 and $95.5 \%$, respectively. The AUC of EHHADH was $0.532(95 \%$ CI, 0.481-0.583; $\mathrm{P}=0.421$ ), the cut-off value for EHHADH was 9.78, and the sensitivity and specificity were 26 and $100 \%$, respectively. The AUC of PEX11A was 0.677 (95\% CI, 0.626-0.728; $\mathrm{P}=0.0001)$, the cut-off value for PEX11A was 8.57 , and the sensitivity and specificity were 88.1 and $53.1 \%$, respectively. The AUC of CAMK2G was $0.563(95 \%$ CI, 0.513-0.614; $\mathrm{P}=0.110$ ), the cut-off value for CAMK2G was 11.29 , and the sensitivity and specificity were 41.9 and $89.8 \%$, respectively. The AUC of CAMK2D was $0.516(95 \%$ CI, 0.688-0.468; $\mathrm{P}=0.563)$, the cut-off value for CAMK2D was 11.16 , and the sensitivity and specificity were 96.6 and $30.5 \%$, respectively (Fig. 9A-H; Table V).

\section{Discussion}

Hox genes, which are also known as homeotic genes or homologous genes, are genes that specifically regulate the morphology of living organisms. HOXA13 is a member of the HOX family. In recent years, the role of HOXA13 in cancer has been widely studied $(18,19,21,32,33)$. There are only two reports on the expression of HOXA13 in LUAD. One of these studies focused on the expression of HOTTIP in NSCLC and identified HOTTIP as a transcriptional regulator of HOXA13 in lung cancer cells (26). Ectopic expression of HOTTIP was revealed to decrease the endogenous level of HOXA13, while HOTTIP knockdown increased the expression of HOXA13. This previous study did not focus on HOXA13 and did not consider the expression or clinical significance of HOXA13 in LUAD (25). Another study examined changes in the copy number of genes in early LUAD and obtained evidence indicating that HOXA13 may be an important target gene in the progression of LUAC (26). However, that study focused on gene amplification patterns and on target genes on the short arm of chromosome 7 , and did not use combined case data to investigate changes in HOXA13 expression in LUAD. The present study utilized the LUAD patient sequencing data in the public TCGA database and for the first time revealed that the expression level of HOXA13 in LUAD tissues is significantly higher than that in non-cancerous lung tissues. The results were validated using the Oncomine online database and RT-qPCR data obtained from clinical samples. In order to obtain a comprehensive result, a meta-analysis was performed. The results of this fully demonstrated that HOXA13 has a markedly increased trend in LUAD. The expression data of HOXA13 in 191 different lung cancer cell lines were also collected from the CCLE online database for further verification of these conclusions. In addition, bioinformatics were used to study the mechanism by which HOXA13 promotes malignant biological behavior in LUAD occurrence and development, and revealed that HOXA13 promotes the progression of LUAD by controlling the expression of a series of genes that induce changes in certain important pathways. These results suggested that HOXA13 serves a vital role in the development and progression of LUAD and that HOXA13 may have clinical potential.

The present study integrated data from a variety of sources, and the different detection methods used all suggested that HOXA13 is overexpressed in LUAD. Analysis of the TCGA data revealed that HOXA13 was expressed at a significantly higher level in cancerous tissues than in 


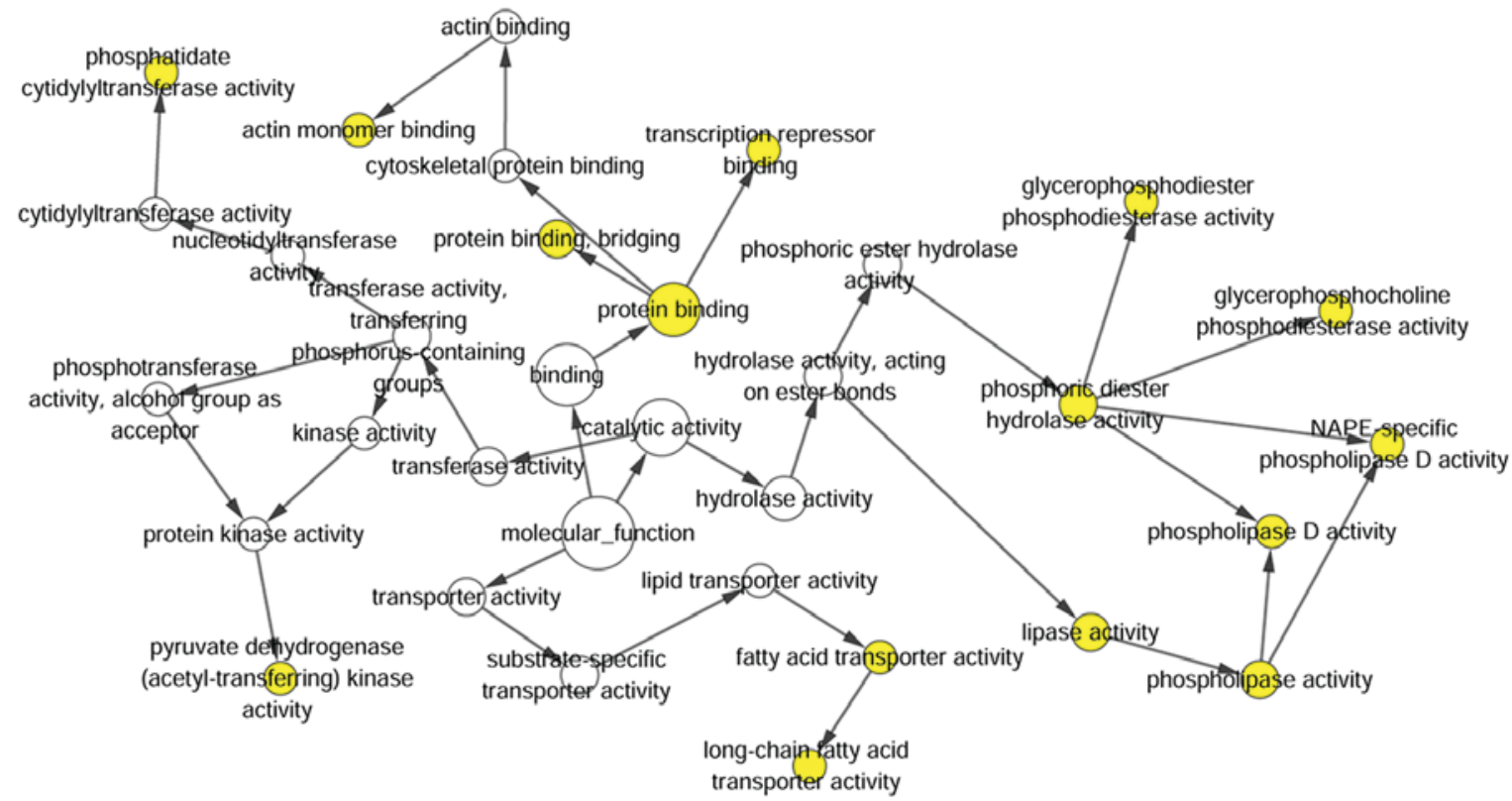

Figure 5. Gene Ontology analysis of Molecular Function category analysis of HOXA13-related genes. Each circle represents a molecular function in which related genes of HOXA13 participate.

A

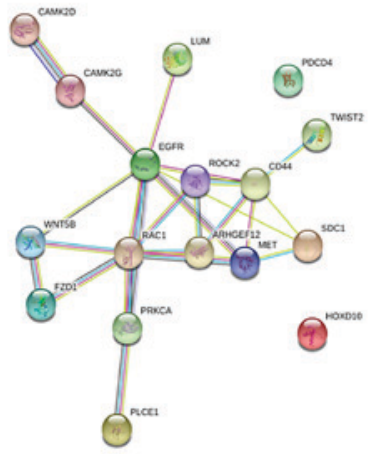

C

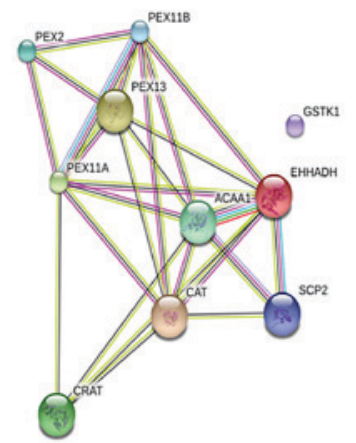

B

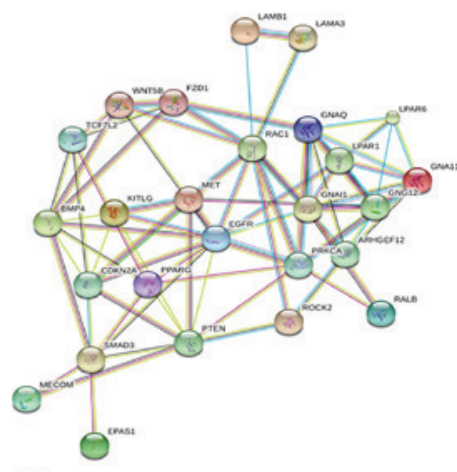

D

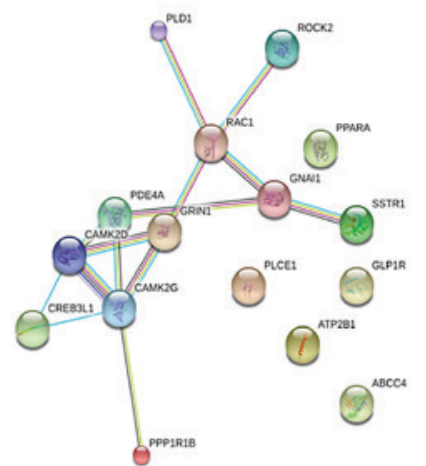

Figure 6. Protein-protein interaction analysis of HOXA13-related genes in the first four Kyoto Encyclopedia of Genes and Genomes pathways. Each node represents a different gene. Each line represents a connection between two different genes: (A) Proteoglycans in cancer ( $\mathrm{P}=0.00140)$; (B) Pathways in cancer $(\mathrm{P}=0.00205) ;(\mathrm{C})$ Peroxisome $\mathrm{P}=0.00209)$; and $(\mathrm{D})$ cAMP signaling pathway $(\mathrm{P}=0.00334)$.

adjacent non-cancerous tissues. ROC curve analysis was used to demonstrate that a HOXA13 expression level of $>1.27$ suggested that the diagnosis was LUAD (AUC $=0.839$; $\mathrm{P}=0.001)$. This result indicated that HOXA13 serves a role in the development of LUAD and that it may be a prospective molecular biomarker for the diagnosis of LUAD. Oncomine was also used to validate these results. Among the four sets of data obtained from Oncomine, the expression values of HOXA13 in LUAD tissues were higher than those in normal tissues for the Hou $(\mathrm{P}=0.058)$ and Selamat $(\mathrm{P}=0.002)$ datasets, 
Table IV. HOXA13-related signaling pathways in lung adenocarcinoma. The top ten significant pathways in KEGG and GO.

\begin{tabular}{|c|c|c|c|c|c|}
\hline Category & ID & GO terms & Count & $\%$ & P-value \\
\hline KEGG_PATHWAY & hsa05205 & Proteoglycans in cancer & 17 & 0.01558 & 0.00140 \\
\hline KEGG_PATHWAY & hsa05200 & Pathways in cancer & 26 & 0.02383 & 0.00205 \\
\hline KEGG_PATHWAY & hsa04146 & Peroxisome & 10 & 0.00917 & 0.00209 \\
\hline KEGG_PATHWAY & hsa04024 & cAMP signaling pathway & 16 & 0.01467 & 0.00334 \\
\hline KEGG_PATHWAY & hsa00920 & Sulfur metabolism & 4 & 0.00367 & 0.00405 \\
\hline KEGG_PATHWAY & hsa04728 & Dopaminergic synapse & 12 & 0.01100 & 0.00448 \\
\hline KEGG_PATHWAY & hsa04070 & Phosphatidylinositol signaling system & 10 & 0.00917 & 0.00641 \\
\hline KEGG_PATHWAY & hsa04916 & Melanogenesis & 10 & 0.00917 & 0.00730 \\
\hline KEGG_PATHWAY & hsa04921 & Oxytocin signaling pathway & 13 & 0.01192 & 0.00816 \\
\hline KEGG_PATHWAY & hsa04020 & Calcium signaling pathway & 14 & 0.01283 & 0.00865 \\
\hline GOTERM_CC_DIRECT & GO:0070062 & Extracellular exosome & 154 & 0.14116 & $2.47 \mathrm{E}-09$ \\
\hline GOTERM_CC_DIRECT & GO:0005794 & Golgi apparatus & 60 & 0.05500 & 4.53E-07 \\
\hline GOTERM_CC_DIRECT & GO:0000139 & Golgi membrane & 45 & 0.04125 & $1.54 \mathrm{E}-06$ \\
\hline GOTERM_CC_DIRECT & GO:0016020 & Membrane & 117 & 0.10724 & $1.81 \mathrm{E}-06$ \\
\hline GOTERM_CC_DIRECT & GO:0005783 & Endoplasmic reticulum & 52 & 0.04766 & 4.65E-05 \\
\hline GOTERM_CC_DIRECT & GO:0005789 & Endoplasmic reticulum membrane & 52 & 0.04766 & $1.34 \mathrm{E}-04$ \\
\hline GOTERM_CC_DIRECT & GO:0016324 & Apical plasma membrane & 24 & 0.02200 & $2.08 \mathrm{E}-04$ \\
\hline GOTERM_CC_DIRECT & GO:0005777 & Peroxisome & 13 & 0.01191 & $2.49 \mathrm{E}-04$ \\
\hline GOTERM_CC_DIRECT & GO:0048471 & Perinuclear region of cytoplasm & 40 & 0.03667 & $2.49 \mathrm{E}-04$ \\
\hline GOTERM_CC_DIRECT & GO:0005829 & Cytosol & 149 & 0.13657 & 3.92E-04 \\
\hline GOTERM_MF_DIRECT & GO:0043565 & Sequence-specific DNA binding & 44 & 0.04033 & $1.22 \mathrm{E}-07$ \\
\hline GOTERM_MF_DIRECT & GO:0003707 & Steroid hormone receptor activity & 9 & 0.00825 & $6.64 \mathrm{E}-04$ \\
\hline GOTERM_MF_DIRECT & GO:0005096 & GTPase activator activity & 22 & 0.02017 & 7.84E-04 \\
\hline GOTERM_MF_DIRECT & GO:0005515 & Protein binding & 343 & 0.31440 & 0.00120 \\
\hline GOTERM_MF_DIRECT & GO:0000978 & $\begin{array}{l}\text { RNA polymerase II core promoter proximal } \\
\text { region sequence-specific DNA binding }\end{array}$ & 25 & 0.02292 & 0.00154 \\
\hline GOTERM_MF_DIRECT & GO:0005509 & Calcium ion binding & 41 & 0.03758 & 0.00227 \\
\hline GOTERM_MF_DIRECT & GO:0017137 & Rab GTPase binding & 13 & 0.01192 & 0.00249 \\
\hline GOTERM_MF_DIRECT & GO:0019902 & Phosphatase binding & 7 & 0.00642 & 0.00443 \\
\hline GOTERM_MF_DIRECT & GO:0008134 & Transcription factor binding & 20 & 0.01833 & 0.00509 \\
\hline GOTERM_MF_DIRECT & GO:0000287 & Magnesium ion binding & 16 & 0.01467 & 0.00522 \\
\hline GOTERM_BP_DIRECT & GO:0009952 & Anterior/posterior pattern specification & 14 & 0.01283 & $3.74 \mathrm{E}-06$ \\
\hline GOTERM_BP_DIRECT & GO:0043401 & $\begin{array}{l}\text { Steroid hormone- mediated signaling } \\
\text { pathway }\end{array}$ & 11 & 0.01010 & $2.48 \mathrm{E}-05$ \\
\hline GOTERM_BP_DIRECT & GO:0001657 & Ureteric bud development & 9 & 0.00825 & 4.11E-05 \\
\hline GOTERM_BP_DIRECT & GO:0035115 & Embryonic forelimb morphogenesis & 8 & 0.00733 & $9.67 \mathrm{E}-05$ \\
\hline GOTERM_BP_DIRECT & GO:0043547 & Positive regulation of GTPase activity & 38 & 0.03483 & 1.97E-04 \\
\hline GOTERM_BP_DIRECT & GO:0045944 & $\begin{array}{l}\text { Positive regulation of transcription from } \\
\text { RNA polymerase II promoter }\end{array}$ & 57 & 0.05225 & $2.02 \mathrm{E}-04$ \\
\hline GOTERM_BP_DIRECT & GO:0007031 & Peroxisome organization & 6 & 0.00550 & $3.01 \mathrm{E}-04$ \\
\hline GOTERM_BP_DIRECT & GO:0042733 & Embryonic digit morphogenesis & 9 & 0.00825 & $6.87 \mathrm{E}-04$ \\
\hline GOTERM_BP_DIRECT & GO:0045892 & $\begin{array}{l}\text { Negative regulation of transcription, } \\
\text { DNA-templated }\end{array}$ & 33 & 0.03025 & 7.35E-04 \\
\hline GOTERM_BP_DIRECT & GO:0051216 & Cartilage development & 9 & 0.00825 & 9.79E-04 \\
\hline
\end{tabular}

GO, Gene Ontology; KEGG, Kyoto Encyclopedia of Genes and Genomes; BP, biological process; CC, cellular component; MF, molecular function.

and the difference in the Selamat dataset was statistically significant. RT-qPCR was used to examine 29 LUAD tissues and 29 corresponding non-cancerous tissues. Although the expression of HOXA13 in the LUAD tissues tended to be increased, compared with that in the non-cancerous control tissues, the difference was not statistically significant. The 
A

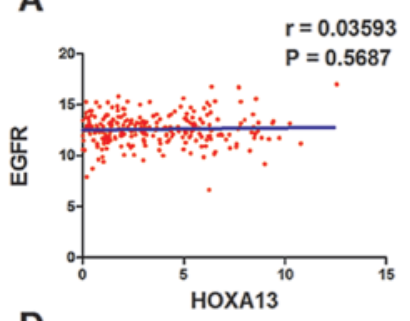

D

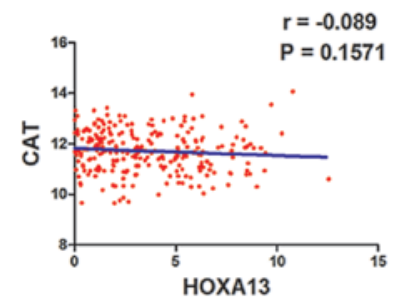

G

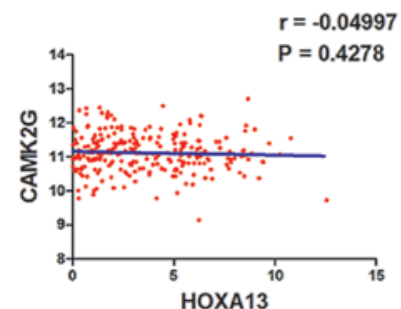

B

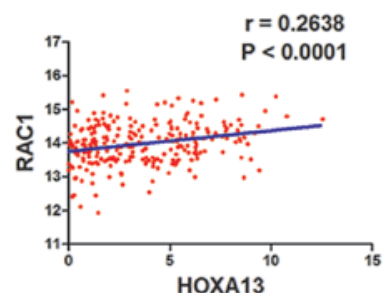

E

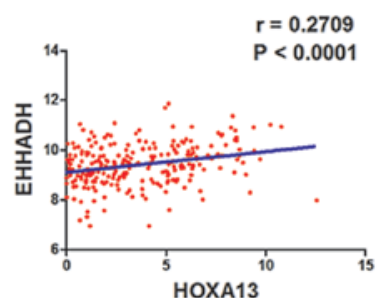

$\mathrm{H}$

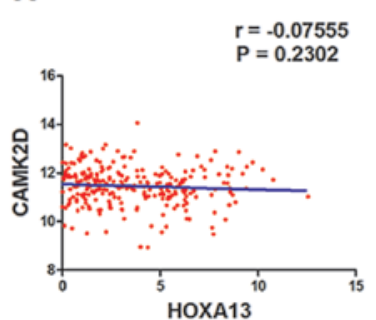

C

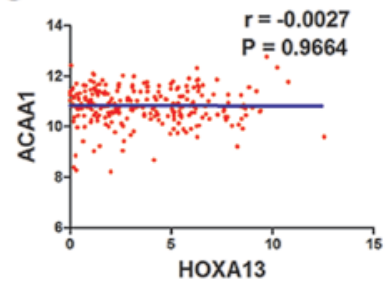

$\mathrm{F}$

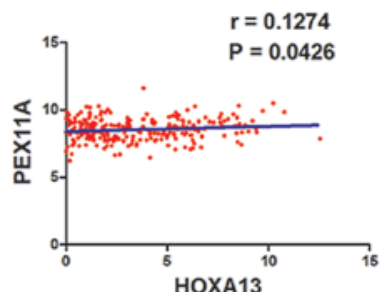

Figure 7. Correlation between the expression of HOXA13 and that of hub genes. (A) Correlations between HOXA13 and EGFR; (B) correlations between HOXA13 and RAC1; (C) correlations between HOXA13 and ACAA1; (D) correlations between HOXA13 and CAT; (E) correlations between HOXA13 and EHHADH; (F) correlations between HOXA13 and PEX11A; (G) correlations between HOXA13 and CAMK2G; and (H) correlations between HOXA13 and CAMK2D. EGFR, epidermal growth factor receptor; RAC1, Rac family small GTPase 1; ACAA1, acetyl-CoA acyltransferase 1; CAT, catalase; EHHADH, enoyl-CoA hydratase and 3-hydroxyacyl CoA dehydrogenase; PEX11A, peroxisomal biogenesis factor 11 $\alpha$; CAMK2, calcium/calmodulin-dependent protein kinase II.

A

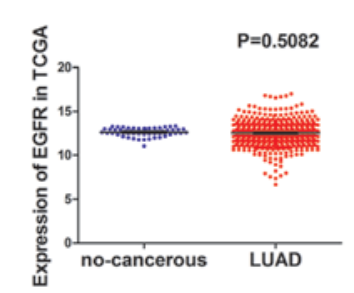

D

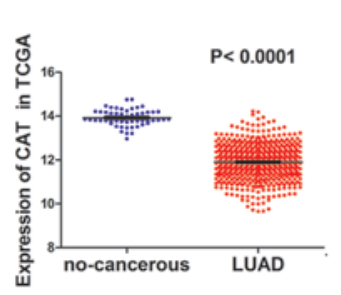

G

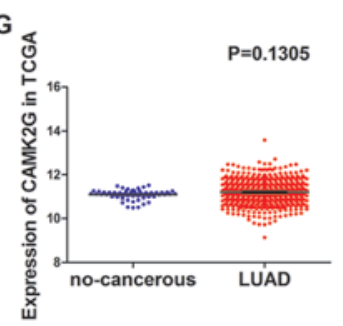

B
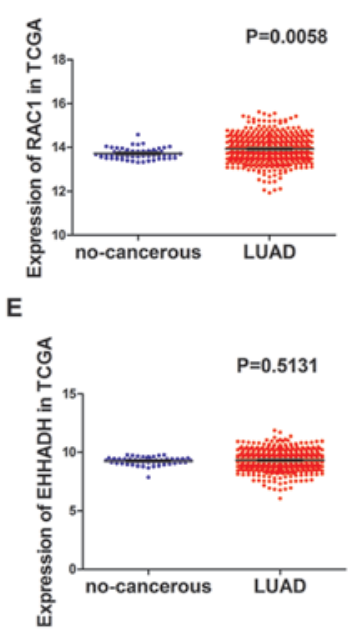

H

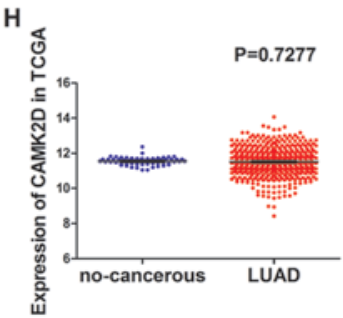

C

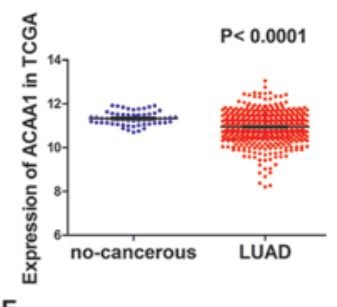

$\mathbf{F}$

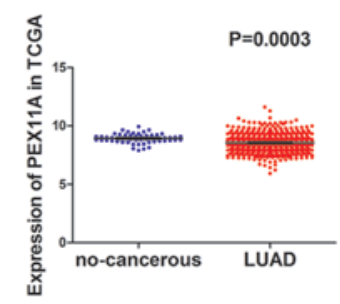

Figure 8. The expression value of hub genes in the LUAD group and the non-cancerous group in TCGA. (A) EGFR, (B) RAC1, (C) ACAA1, (D) CAT, (E) EHHADH, (F) PEX11A, (G) CAMK2G and (H) CAMK2D (normal, 59 cases; lung adenocarcinoma, 535 cases). LUAD, lung adenocarcinoma; TCGA, The Cancer Genome Atlas; EGFR, epidermal growth factor receptor; RAC1, Rac family small GTPase 1; ACAA1, acetyl-CoA acyltransferase 1; CAT, catalase; EHHADH, enoyl-CoA hydratase and 3-hydroxyacyl CoA dehydrogenase; PEX11A, peroxisomal biogenesis factor 11 $\alpha$; CAMK2, calcium/calmodulin-dependent protein kinase II. 

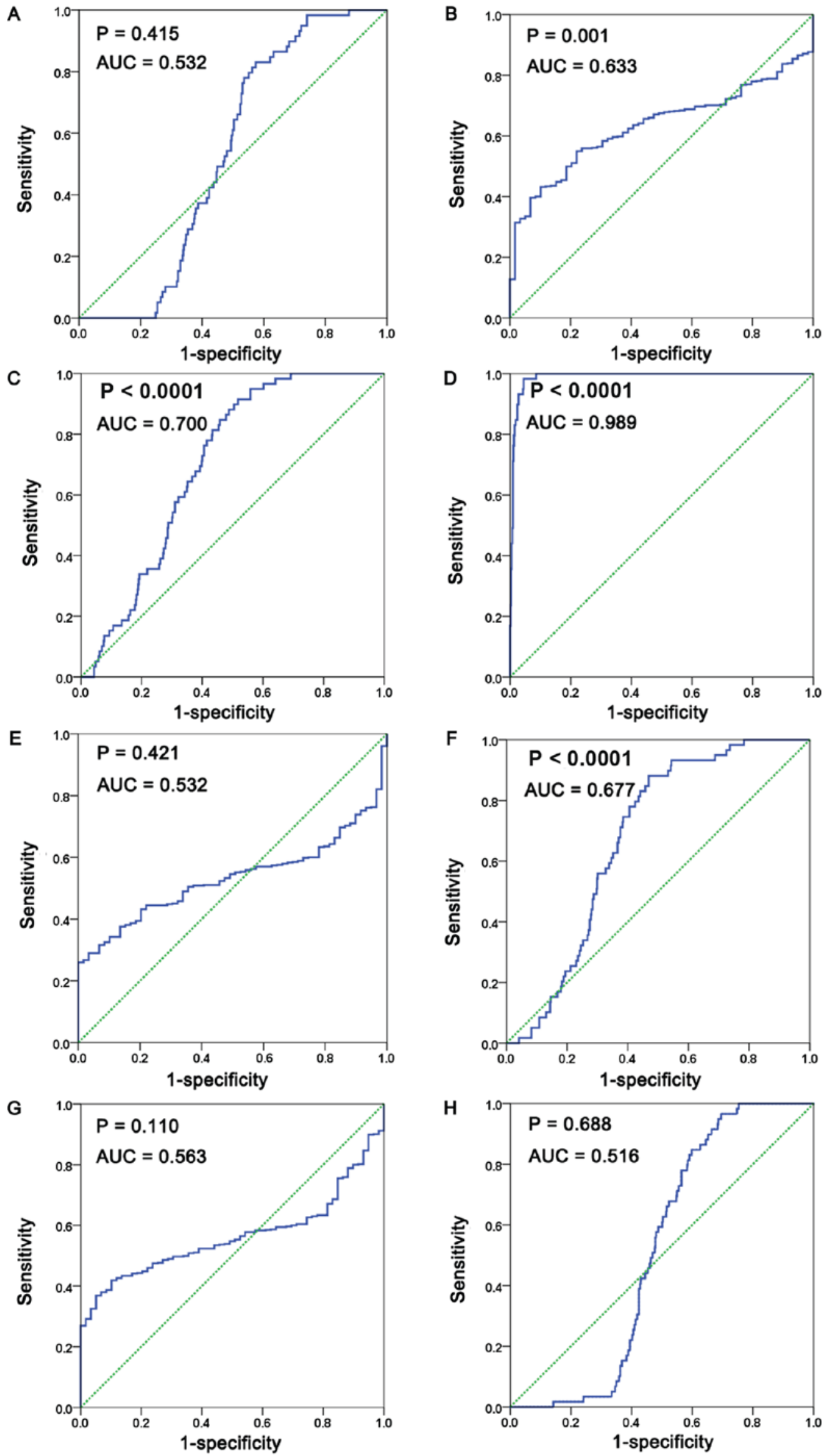

Figure 9. ROC curve for discrimination of LUAD from non-cancerous cases. ROC curve of (A) EGFR, (B) RAC1, (C) ACAA1, (D) CAT, (E) EHHADH, (F) PEX11A, (G) CAMK2G and (H) CAMK2D. (normal, 59 cases; lung adenocarcinoma, 535 cases). ROC, receiver operating characteristic; LUAD, lung adenocarcinoma; EGFR, epidermal growth factor receptor; RAC1, Rac family small GTPase 1; ACAA1, acetyl-CoA acyltransferase 1; CAT, catalase; EHHADH, enoyl-CoA hydratase and 3-hydroxyacyl CoA dehydrogenase; PEX11A, peroxisomal biogenesis factor 11 $\alpha$; CAMK2, calcium/calmodulin-dependent protein kinase II. 
reason for this may be that the number of cases analyzed was small. In order to acquire a comprehensive understanding of the clinical role of HOXA13 in LUAD, a meta-analysis was performed based on all cases from the TCGA database, the in-house RT-qPCR data and data from Oncomine, which included 189 non-cancerous lung tissues and 637 LUAD cases. The total SMD reached 0.346 (95\% CI, 0.052-0.640). SMD $>0$ and its $95 \%$ CI did not cross the 0 value, which indicated that the expression of HOXA13 in tumor tissues was markedly higher than that in the non-cancerous lung tissues. The pooled sensitivity was 0.64 and the pooled specificity was 0.77 . Furthermore, the results of the meta-analyses revealed that the DOR for HOXA13 in LUAD diagnosis was 6.05 and that the AUC was 0.78 , indicating the variation in HOXA13 expression between non-cancerous and cancerous lung tissues $(34,35)$. Additionally, in 192 lung cancer cell lines, distinct expression levels of HOXA13 were observed. These results demonstrated that HOXA13 expression is markedly increased in LUAD. Therefore, HOXA13 may be involved in the development of LUAD, but the results of the present study require validation by other independent cohorts and other detection methods.

The aforementioned results suggested that HOXA13 expression is associated with the development of LUAD, but the molecular mechanism of HOXA13 action remains unknown. Therefore, the present study attempted to predict the potential mechanism of HOXA13 action in LUAD using bioinformatics methods. To begin with, 679 genes associated with HOXA13 were identified through the cBioPortal and MEM databases. GO, KEGG and PPI analyses of these genes were conducted. Significant results were adopted according to the P-value provided by DAVID. It was revealed that these genes were most enriched in the cytoplasm, integral component of membrane, plasma membrane and extracellular exosome, which indicated a potential membrane-associated metabolism regulated by these genes. Furthermore, for biological process, these genes were significantly involved in positive regulation of transcription from RNA polymerase II promoter, signal transduction and positive regulation of GTPase activity, which contribute to RNA translation and transcription, and may be associated with cell proliferation. As for molecular function, the selected genes were significantly involved in protein binding, transcription factor activity and sequence-specific DNA binding, which exhibited a high tendency toward cellular invasion. Furthermore, in KEGG pathway analysis, the targeted genes were most associated with Pathways in cancer, Proteoglycans in cancer and the cAMP signaling pathway, which indicated that the potential HOXA13 co-expression genes may participate in the development of tumorigenesis in LUAD.

To further determine the role of HOXA13 in the occurrence and development of LUAD, the genes that were most highly enriched in the four KEGG pathways were selected for PPI network analysis. The hub genes obtained from the four PPI networks included eight genes: EGFR, RAC1, ACAA1, CAT, EHHADH, PEX11A, CAMK2G and CAMK2D. EGFR and RAC1 were revealed to be hub genes in PPI analysis of two pathways and are therefore particularly notable as co-expression genes for HOXA13. Among the eight genes, RAC1, EHHADH and PEX11A were significantly correlated

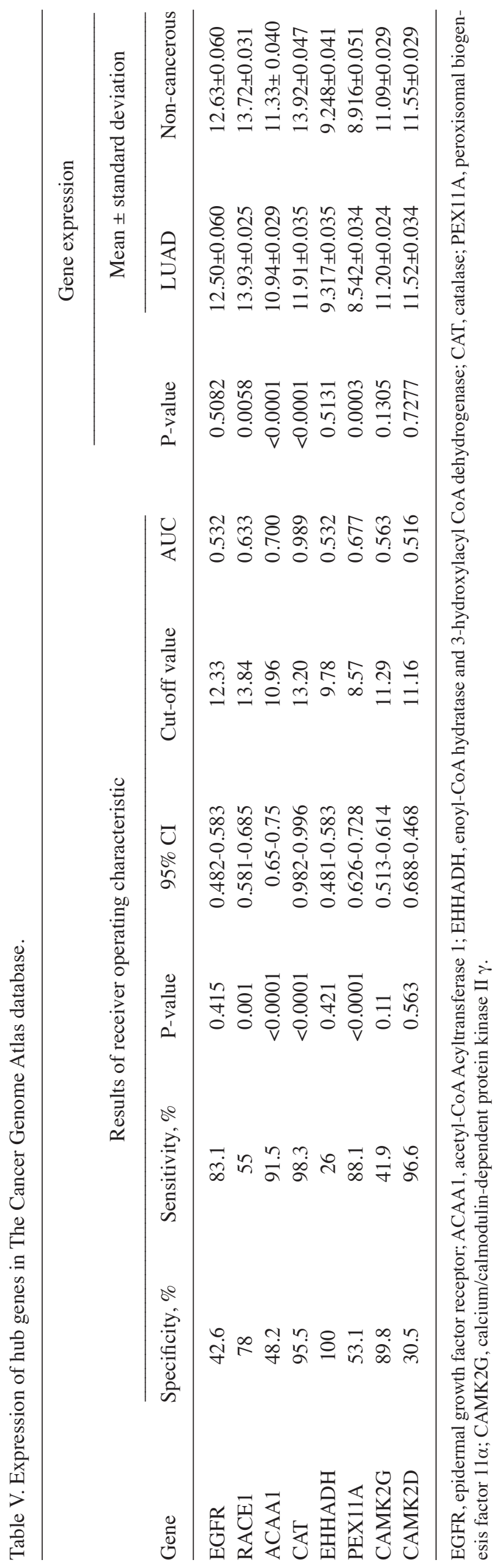


with HOXA13, and ACAA1, CAT, CAMK2G, CAMK2D and EGFR were not significantly correlated with HOXA13. The present study further investigated the association between expression of hub genes in normal tissues and LUAD tissues based on the data from the TCGA database, and the results revealed that 4 of the 8 hub genes, RAC1, CAT, EHHADH and PEX11A, exhibited a significant difference in expression of HOXA13 between non-cancerous tissues and LUAD tissues. Additionally, ROC curves of these four hub genes exhibited larger AUCs, with statistically significant differences between non-cancerous tissues and LUAD tissues $(\mathrm{P}<0.05)$. Notably, two of these four genes, RAC1 $(\mathrm{P}<0.0001)$ and PEX11A $(\mathrm{P}=0.0426)$, have a relatively clearer correlation with HOXA13, compared with the others. This demonstrates that RAC1, a G protein, is a signal molecular switch that regulates a variety of cell activities and gene expression. RAC1 is involved in the modulation of phagocytosis, adhesion, cell movement, cell proliferation and axon formation (36-39). RAC1 serves a pivotal role in cancer angiogenesis, invasion and metastasis $(40,41)$. There are ongoing in-depth studies on lung cancer clinical treatment using $\mathrm{RAC} 1$ as the target $(39,42)$. The role of PEX11A and its association with cancer has not yet been reported. The results of the bioinformatics analysis in the present study demonstrated that HOXA13 may influence the expression of these hub genes in such a way as to promote the occurrence and development of LUAD. However, this hypothesis requires validation in future in vitro and in vivo experiments.

There are certain limitations to the present study. To begin with, the number of cases used for the investigation of HOXA13 was small and the results require validation in future studies with larger sample sizes. Additionally, the mechanism of HOXA13 was predicted and identification of the specific mechanism will require further in vitro and in vivo validation.

In conclusion, the expression of HOXA13 in patients with LUAD and its potential clinical value were analyzed comprehensively in the present study using data from a variety of sources. Bioinformatics analysis was used to obtain evidence that HOXA13 may promote the occurrence and development of LUAD.

\section{Acknowledgements}

Not applicable.

\section{Funding}

The present study was supported by the funds of the National Natural Science Foundation of China (grant nos. 81560469 and 81360327), the Natural Science Foundation of Guangxi, China (grant no.2015GXNSFCA139009 and 2017GXNSFAA198016) and the Guangxi University Student Innovative Plan (grant nos. 201710598001 and 201710598080).

\section{Availability of data and materials}

The datasets used and/or analyzed during the current study are available from the corresponding author on reasonable request.

\section{Authors' contributions}

YD and RQH conceived and designed the study. $Y D, R Q H$, $\mathrm{RZ}$ and $\mathrm{BLG}$ performed the experiments. $\mathrm{YD}$ and $\mathrm{RQH}$ wrote the paper. GC, XHH and YZ made substantial contributions to the design of the current study, acquisition of data, interpretation of data and revising the manuscript. All authors read and approved the final manuscript.

\section{Ethics statement and consent to participate}

Not applicable.

\section{Consent for publication}

Not applicable.

\section{Competing interests}

The authors declare that they have no competing interests.

\section{References}

1. Chen N, Yang X, Guo W, You J, Wu Q, Zhang G, Li H, Geng D, Jin T, Fu J and Zhang Y: Association of polymorphisms in the telomere-related gene ACYP2 with lung cancer risk in the Chinese Han population. Oncotarget 7: 87473-87478, 2016.

2. Huang C, Liu S, Wang H, Zhang Z, Yang Q and Gao F: LncRNA PVT1 overexpression is a poor prognostic biomarker and regulates migration and invasion in small cell lung cancer. Am J Transl Res 8: 5025-5034, 2016.

3. Shen Y, Tian Z, Lu D, Huang J, Zhang Z, Li X and Li J: Impact of pneumonia and lung cancer on mortality of women with hypertension. Sci Rep 6: 20, 2016.

4. Shahid M, Choi TG, Nguyen MN, Matondo A, Jo YH, Yoo JY, Nguyen NN, Yun HR, Kim J, Akter S, et al: An 8-gene signature for prediction of prognosis and chemoresponse in non-small cell lung cancer. Oncotarget 7: 86561-86572, 2016.

5. Xing P, Wang S, Hao X, Zhang T and Li J: Clinical data from the real world: Efficacy of Crizotinib in Chinese patients with advanced ALK-rearranged non-small cell lung cancer and brain metastases. Oncotarget 7: 84666-84674, 2016.

6. Liu K, Chen HL, Gu MM and You QS: A three gene-based risk score predicts prognosis of resected non-small-cell lung cancer. Int J Clin Exp Pathol 8: 16081-16088, 2015.

7. Yazawa T, Kaira K, Shimizu K, Shimizu A, Mori K, Nagashima T, Ohtaki Y, Oyama T, Mogi A and Kuwano H: Prognostic significance of $\beta 2$-adrenergic receptor expression in non-small cell lung cancer. Am J Transl Res 8: 5059-5070, 2016.

8. Chiba M, Togashi Y, Tomida S, Mizuuchi H, Nakamura Y, Banno E, Hayashi H, Terashima M, De Velasco MA, Sakai K, et al: MEK inhibitors against MET-amplified non-small cell lung cancer. Int J Oncol 49: 2236-2244, 2016.

9. Kotsakis A, Koinis F, Katsarou A, Gioulbasani M, Aggouraki D, Kentepozidis N, Georgoulias V and Vetsika EK: Prognostic value of circulating regulatory $\mathrm{T}$ cell subsets in untreated non-small cell lung cancer patients. Sci Rep 6: 39247, 2016.

10. Lingzi X, Zhihua Y, Xuelian L, Yangwu R, Haibo Z, Yuxia Z and Baosen Z: Genetic variants in microRNAs predict non-small cell lung cancer prognosis in Chinese female population in a prospective cohort study. Oncotarget 7: 83101-83114, 2016.

11. Song J, Liu Z, Zhong W, Huang Y, Ma Z, Dong D, Liang C and Tian J: Non-small cell lung cancer: Quantitative phenotypic analysis of CT images as a potential marker of prognosis. Sci Rep 6: 38282, 2016.

12. Tang W, Han M, Ruan B, Jin W, Lou J, Yuan X, Chen D, Chen Y, Shin VY, Jin H and Wang X: Overexpression of GOLPH3 is associated with poor survival in Non-small-cell lung cancer. Am J Transl Res 8: 1756-1762, 2016.

13. Li D and He S: Pemetrexed and cyclophosphamide combination therapy for the treatment of non-small cell lung cancer. Int J Clin Exp Pathol 8: 14693-14700, 2015. 
14. Xia X, Lu JJ, Zhang SS, Su CH and Luo HH: Midkine is a serum and urinary biomarker for the detection and prognosis of non-small cell lung cancer. Oncotarget 7: 87462-87472, 2016.

15. Kondo M, Yamamoto T, Takahashi S and Taira M: Comprehensive analyses of hox gene expression in Xenopus laevis embryos and adult tissues. Dev Growth Differ 59: 526-539, 2017.

16. Bhatlekar S, Fields JZ and Boman BM: HOX genes and their role in the development of human cancers. J Mol Med (Berl) 92: 811-823, 2014

17. Wu DC, Wang SSW, Liu CJ, Wuputra K, Kato K, Lee YL, Lin YC, Tsai MH, Ku CC, Lin WH, et al: Reprogramming Antagonizes the Oncogenicity of HOXA13-Long Noncoding RNA HOTTIP Axis in gastric cancer cells. Stem Cells 35: 2115-2128, 2017.

18. Chang S, Liu J, Guo S, He S, Qiu G, Lu J, Wang J, Fan L, Zhao W and Che X: HOTTIP and HOXA13 are oncogenes associated with gastric cancer progression. Oncol Rep 35: 3577-3585, 2016.

19. Duan R, Han L, Wang Q, Wei J, Chen L, Zhang J, Kang C and Wang L: HOXA13 is a potential GBM diagnostic marker and promotes glioma invasion by activating the Wnt and TGF- $\beta$ pathways. Oncotarget 6: 27778-27793, 2015.

20. Dong Y, Cai Y, Liu B, Jiao X, Li ZT, Guo DY, Li XW, Wang YJ and Yang DK: HOXA13 is associated with unfavorable survival and acts as a novel oncogene in prostate carcinoma. Future Oncol 13: 1505-1516, 2017.

21. Wang SS, Wuputra K, Liu CJ, Lin YC, Chen YT, Chai CY, Lin CS, Kuo KK, Tsai MH, Wang SW, et al: Oncogenic function of the homeobox A13-long noncoding RNA HOTTIP-insulin growth factor-binding protein 3 axis in human gastric cancer. Oncotarget 7: 36049-36064, 2016.

22. Chandrasekaran G, Hwang EC, Kang TW, Kwon DD, Park K, Lee JJ and Lakshmanan VK: Computational Modeling of complete HOXB13 protein for predicting the functional effect of SNPs and the associated role in hereditary prostate cancer. Sci Rep 7: 43830, 2017.

23. Saha SS, Chowdhury RR, Mondal NR, Roy S and Sengupta S: Expression signatures of HOX cluster genes in cervical cancer pathogenesis: Impact of human papillomavirus type 16 oncoprotein E7. Oncotarget 8: 36591-36602, 2017.

24. Kelly Z, Moller-Levet C, McGrath S, Butler-Manuel S, Kavitha Madhuri T, Kierzek AM, Pandha H, Morgan R and Michael A: The prognostic significance of specific HOX gene expression patterns in ovarian cancer. Int J Cancer 139: 1608-1617, 2016

25. Sang Y, Zhou F, Wang D, Bi X, Liu X, Hao Z, Li Q and Zhang W: Up-regulation of long non-coding HOTTIP functions as an oncogene by regulating HOXA13 in non-small cell lung cancer. Am J Transl Res 8: 2022-2032, 2016.

26. Kang JU: Characterization of amplification patterns and target genes on the short arm of chromosome 7 in early-stage lung adenocarcinoma. Mol Med Rep 8: 1373-1378, 2013.

27. Hou J, Aerts J, den Hamer B, van Ijcken W, den Bakker M, Riegman P, van der Leest C, van der Spek P, Foekens JA, Hoogsteden $\mathrm{HC}$, et al: Gene expression-based classification of non-small cell lung carcinomas and survival prediction. PLoS One 5: 1932-6203, 2010.

28. Selamat SA, Chung BS, Girard L, Zhang W, Zhang Y, Campan M, Siegmund KD, Koss MN, Hagen JA, Lam WL, et al: Genome-scale analysis of DNA methylation in lung adenocarcinoma and integration with mRNA expression. Genome Res 22: $1197-1211,2012$.

29. Garber ME, Troyanskaya OG, Schluens K, Petersen S, Thaesler Z, Pacyna-Gengelbach M, van de Rijn M, Rosen GD, Perou CM, Whyte RI, et al: Diversity of gene expression in adenocarcinoma of the lung. Proc Natl Acad Sci USA 98: 13784-13789, 2001
30. Okayama H, Kohno T, Ishii Y, Shimada Y, Shiraishi K, Iwakawa R, Furuta K, Tsuta K, Shibata T, Yamamoto S, et al: Identification of genes upregulated in ALK-positive and EGFR/KRAS/ALK-negative lung adenocarcinomas. Cancer Res 72: 100-111, 2012.

31. Chen G, Kronenberger P, Umelo IA, Teugels E and De Grève J: Quantification of epidermal growth factor receptor T790M mutant transcripts in lung cancer cells by real-time reverse transcriptase-quantitative polymerase chain reaction. Anal Biochem 398: 266-268, 2010.

32. Guo B, Che T, Shi B, Guo L, Zhang Z, Li L, Cai C and Chen Y: Interaction network analysis of differentially expressed genes and screening of cancer marker in the urine of patients with invasive bladder cancer. Int J Clin Exp Med 8: 3619-3628, 2015.

33. Cheng Y, Jutooru I, Chadalapaka G, Corton JC and Safe S: The long non-coding RNA HOTTIP enhances pancreatic cancer cell proliferation, survival and migration. Oncotarget 6: 10840-10852, 2015.

34. Glas AS, Lijmer JG, Prins MH, Bonsel GJ and Bossuyt PM: The diagnostic odds ratio: A single indicator of test performance. J Clin Epidemiol 56: 1129-1135, 2003.

35. Walter SD: Properties of the summary receiver operating characteristic (SROC) curve for diagnostic test data. Stat Med 21: 1237-1256, 2002

36. Zeng N, Salker MS, Zhang S, Singh Y, Shi B, Stournaras C and Lang F: $1 \alpha, 25(\mathrm{OH}) 2 \mathrm{D} 3$ induces actin depolymerization in endometrial carcinoma cells by targeting RAC1 and PAK1. Cell Physiol Biochem 40: 1455-1464, 2016.

37. Raja R, Sahasrabuddhe NA, Radhakrishnan A, Syed N, Solanki HS, Puttamallesh VN, Balaji SA, Nanjappa V, Datta KK, Babu N, et al: Chronic exposure to cigarette smoke leads to activation of p21 (RAC1)-activated kinase 6 (PAK6) in non-small cell lung cancer cells. Oncotarget 7: 61229-61245, 2016.

38. Wang M, Dong Q and Wang Y: Rab23 is overexpressed in human astrocytoma and promotes cell migration and invasion through regulation of Rac1. Tumour Biol 37: 11049-11055, 2016.

39. Li Z, Guo C, Liu X, Zhou C, Zhu F, Wang X, Wang Q, Shi Y, Wang J, Zhao W and Zhang L: TIPE2 suppresses angiogenesis and non-small cell lung cancer (NSCLC) invasiveness via inhibiting Racl activation and VEGF expression. Oncotarget 7: 62224-62239, 2016.

40. Chen L, DeWispelaere A, Dastvan F, Osborne WR, Blechner C, Windhorst S and Daum G: Smooth Muscle-Alpha actin inhibits vascular smooth muscle cell proliferation and migration by inhibiting Rac1 activity. PLoS One 11: e0155726, 2016.

41. Cho CY, Lee KT, Chen WC, Wang CY, Chang YS, Huang HL, Hsu HP, Yen MC, Lai MZ and Lai MD: MST3 promotes proliferation and tumorigenicity through the VAV2/Rac1 signal axis in breast cancer. Oncotarget 7: 14586-14604, 2016.

42. Zou T, Mao X, Yin J, Li X, Chen J, Zhu T, Li Q, Zhou H and Liu Z: Emerging roles of RAC1 in treating lung cancer patients. Clin Genet 91: 520-528, 2017.

This work is licensed under a Creative Commons Attribution-NonCommercial-NoDerivatives 4.0 International (CC BY-NC-ND 4.0) License. 